\title{
Multi-scale Modeling of Phase Explosion in High Fluence Nanosecond Laser Ablation and Clarification of Ablation Depth Prediction Criterion
}

\author{
Yunfeng Cao and Yung C. Shin ${ }^{1}$ \\ Center for Laser-based Manufacturing, Purdue University \\ West Lafayette, IN 47907
}

\begin{abstract}
When phase explosion occurs, accurate prediction of the ablation behavior in the high energy nanosecond laser ablation process still remains a difficult challenge. In this paper, nanosecond laser ablation of aluminum with phase explosion is investigated through a multi-scale model and experimental verification. The melt ejection behavior during phase explosion is successfully predicted by combined molecular dynamics (MD) and smoothed particle hydrodynamics $(\mathrm{SPH})$ simulations and validated against the experiments. The commonly adopted $0.9 \mathrm{~T}_{\mathrm{c}}$ (critical temperature) criterion for phase explosion boundary is also assessed with the prediction of the ablation depth for both aluminum and copper, and it is found that the $0.9 \mathrm{~T}_{\mathrm{c}}$ criterion does not always work. The multi-scale model developed in this work is shown to have better capability in predicting the ablation behavior when phase explosion is involved.
\end{abstract}

Keywords: phase explosion, nanosecond laser ablation, multiscale modeling

\footnotetext{
${ }^{1}$ Corresponding author: phone: 1-765-494-9775, fax: 1-765-494-0539, email shin@purdue.edu
} 


\section{Introduction}

It is generally known that the laser ablated material consists not only of evaporated atoms, but also particles or droplets formed, either directly by the laser-solid interaction, or later, through condensation (smaller particles condense onto the larger particles), collision between particles, or hydrodynamic sputtering (large particles) in the expanding plume.

In the low laser fluence regime, the particles/droplets are mainly in the nano or submicron scale, which are formed mainly through laser evaporation [1]. A number of models have been reported to describe particle formation and growth in the expanding vapor plume, based on condensation and nucleation theories [2-6]. In general, condensation droplets are typically formed in long-pulse $(\mathrm{ms})$ and low-intensity $\left(10^{4}-10^{5}\right.$ $\mathrm{W} / \mathrm{cm}^{2}$ ) regimes [3]; however, they can also be generated at higher laser intensity and shorter pulse (e.g. $10^{8}-10^{10} \mathrm{~W} / \mathrm{cm}^{2}$ and a few ns pulse) under the slow expansion of the vapor plume into the background gas [7].

With the increase of laser fluence, the surface temperature of a target material may rise close to $90 \%$ of the critical temperature or even higher [8-9]. Under this condition, explosive ejection of liquid melts and droplets will occur and may start dominating the ablation process, which is usually referred to as phase explosion or explosive boiling [9].In this high fluence regime, larger particles/droplets may form with different mechanisms depending on target material and laser conditions. It was proposed based on experimental observation that the vaporized atoms and ions condense as tiny particles on theejected larger droplets, forming an outer layer and even larger particles[10]. For metals, particles are assumed to be formed by liquid (large droplet) ejection, which can 
be the result of several processes. Large droplets can be ejected as a result of transient melting and motion of a liquid caused by steep gradients and the vapor plume recoil pressure [11-14]. The formation of large droplets is assumed to be from the collision between small particles. According to Hergenroder [15], hydrodynamic sputtering may also play a very important role on the large particle formation in a laser ablation process.

At high laser power density, the particle size was measured to have a bimodal shape distribution [15]. Clearly, there are two mechanisms for the particle generation. One is the evaporation induced by laser ablation, which corresponds to the lower peak in the particle size distribution. The other one is due to either condensation, collision between small particles, or hydrodynamic sputtering, which corresponds to the higher peak.

It still remains a difficult challenge to capture the ejected droplets during the material removal by conventional modeling methods (for example, hydrodynamics model) using generated mesh. The mesh size needs to be several times smaller than the ejected molten materials, which requires massive computing resources. Lu [16] proposed a one-dimensional fluid model to describe the thermodynamic evolution during phase explosion. However, Lu's model couldn't predict the melt ejection behavior and the resulting ablation depth. As a walk-around, most researchers use $0.9 \mathrm{~T}_{\mathrm{c}}$ (critical temperature) as the ablation depth prediction criterion when handling phase explosion [17-19]. In the authors' previous work [20], $0.9 \mathrm{~T}_{\mathrm{c}}$ as the ablation depth prediction criterion was shown to yield good accuracy when predicting the ablation depth for aluminum with phase explosion. However, it was found that $0.9 \mathrm{~T}_{\mathrm{c}}$ doesn't work when dealing with a copper target. 
There are some numerical methods proposed in literature to capture these nonlinear phenomena, such as molecular dynamics (MD) and smoothed particle hydrodynamics (SPH). Molecular dynamics (MD) simulation provides an explicit atomistic representation of material heating, vaporization, and plume expansion, and solves problems that cannot be accounted for by continuum models, such as highly nonequilibrium states and fast phase transformations induced by high fluence laser irradiation [21]. The interatomic potential $\mathrm{V}$ is one of the most important parameters governing MD simulation of a certain material, because this potential defines the interactions among atoms that the material consists of and dominates the properties of the material [22]. The force exerted on each atom by other atoms is also determined by the interatomic potential. Therefore, once the interatomic potential is given, MD can be used to simulate many problems with their specific external forces, initial conditions and boundary conditions.

SPH is a meshless computational method represented by a set of particles where each particle moves according to the governing dynamics [23]. In SPH, differential equations are therefore solved by a Lagrangian technique. The basic concept of SPH is that continuous media are represented by discrete particles with volume, density and mass. The particles have a kernel function to define their range of interaction, and the hydrodynamic variables are approximated by integral interpolations. Meshes are not needed in the simulation, which is a major advantage of SPH over Eulerian methods for complex geometries. However, since SPH is a mesoscale method [23]that deals with the particle size in the sub-micron to micron range, SPH alone can't predict the initial 
particle formation in the laser ablation process, where the initial particle size could be far less than 1 micron.

To correctly model the laser ablation process with high laser intensity, particle formation inside the melt pool is simulated by a molecular dynamics model, and the particle movement and the further ejection are described by a smoothed particle hydrodynamic (SPH) model, while all the other parts of the target are modeled by hydrodynamics (HD) equations. Such a coupled HD-MD-SPH model is proposed in this work.

\section{Numerical Model}

A multi-scale model has been developed in this work to tackle the challenges presented earlier. In the initial stage of the laser ablation process, no particle is formed. Therefore the whole domain is described by the HD model. Then in the later stage, some particles will be generated inside the melt pool and the expanding vapor due to either evaporation, condensation (smaller particles condense onto the larger particles), collision between particles, or hydrodynamic sputtering (large particles). At this point, two calculation domains are formed. One is the particle domain (SPH domain) and the other is the HD domain. To obtain the initial particle distribution for the SPH calculation, MD simulation is employed from the beginning of the laser-matter interaction.The HD calculation is based on the model developed earlier in the authors' group [24], while the MD/SPH calculation is conducted with the LAMMPS package [25-26].

Fig. 1 shows the relative position of the MD domain in the multi-scale model, which is right under the laser beam and taken directly from the HD domain. The left and 
right sides of the MD domain are set with periodic boundary conditions. Fig. 2 shows the calculation flow chart for the coupled model. Clearly, the pressure, temperature, and velocity of the interface cells in the HD domain need to be passed to the SPH domain. In Fig.2, the heat flux ( $\left.\mathrm{q}^{\prime \prime}\right)$ at the interface is calculated based on the temperature gradient at the boundary in MD/SPH domain. The force at the interface is calculated based on the pressure gradient at the boundary in HD domain. The mass transfer rate $\mathrm{m}^{\prime}$ is calculated from the mass conservation equation at the interface region.

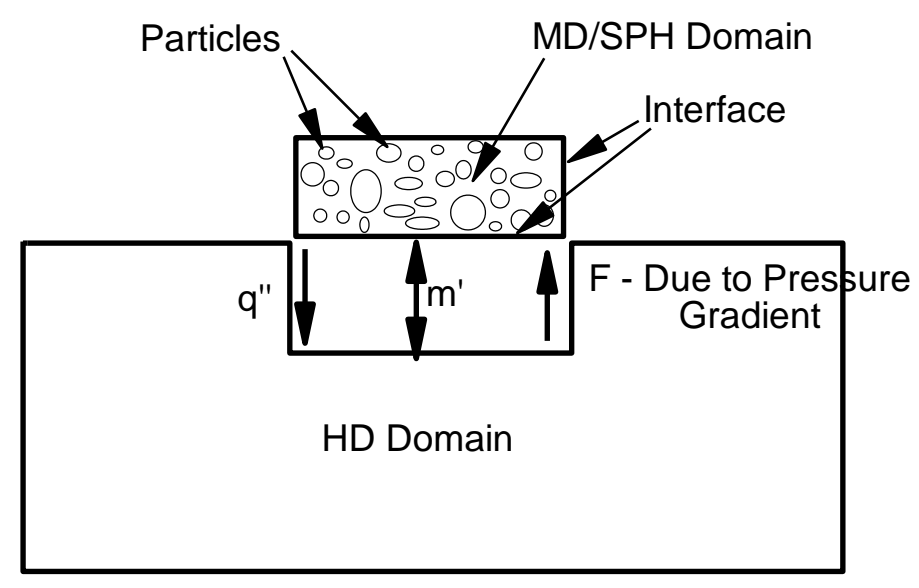

Fig. 1 Calculation Domain

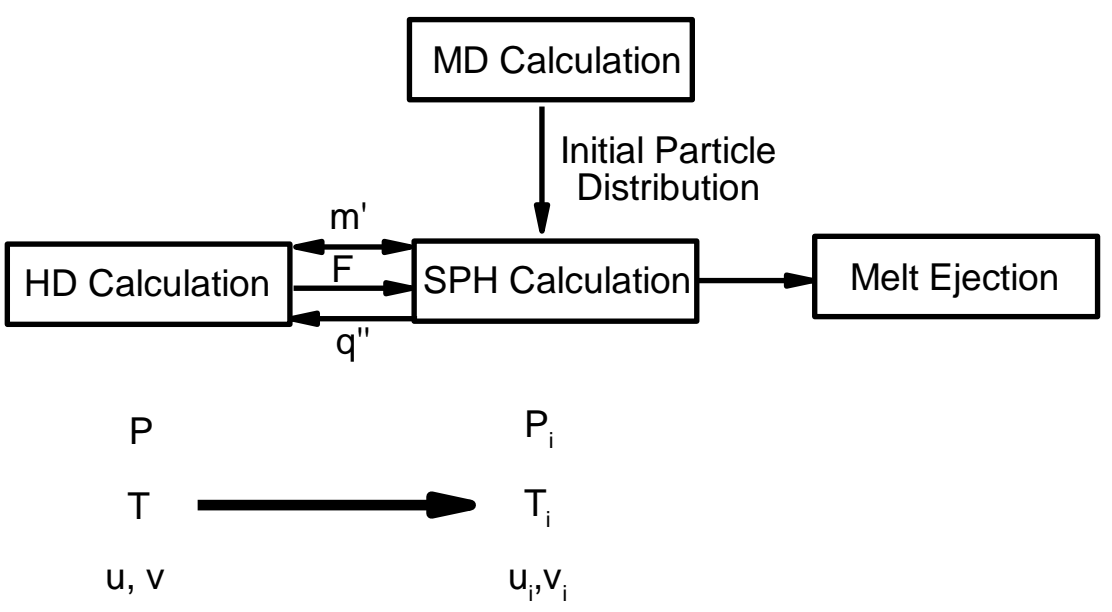

Fig.2 Calculation flow chart for multi-scale model 
In the nanosecond laser ablation process, the laser beam diameter is usually in the range of $100 \mu \mathrm{m}$ to $10 \mathrm{~mm}$, which is much larger than the normal MD simulation size.To consider a larger non-uniform beam used in the experiment as shown in Fig. 3, multiple representative MD cells are taken from the target surface based on the laser intensity distribution to obtain a more accurate initial particle distribution from MD calculation, as seen in Fig. 4. Based on the given beam profile, multiple calculations with different laser fluences are conducted to obtain the particle distribution.

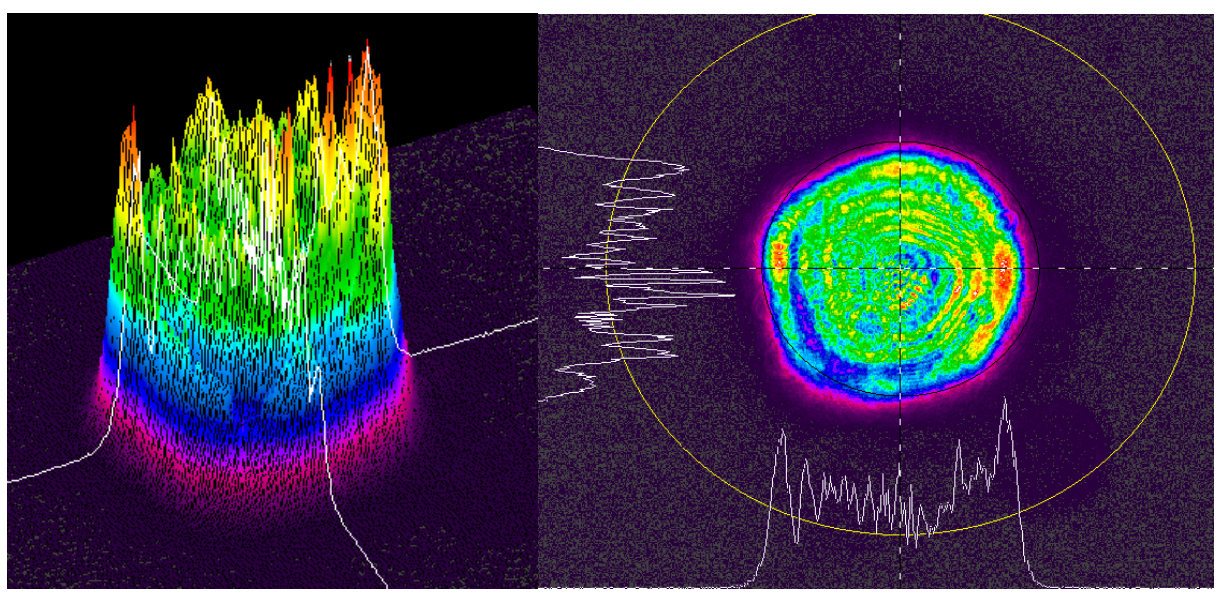

Fig. 3 Beam profile measurement, beam diameter $0.2 \mathrm{~mm}$

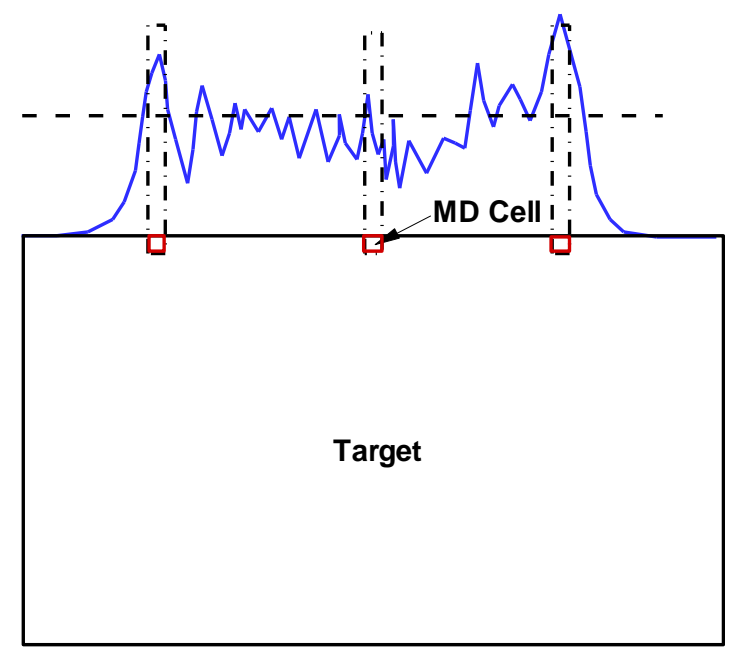

Fig. 4 Laser beam distribution and MD cells 
In the MD calculation, the number of atoms is about 12.5 million in the initial computation domain of $20 \mathrm{~nm} \times 20 \mathrm{~nm} \times 32 \mu \mathrm{m}$. The dimension of the domain is chosen with the consideration of the ablation depth of metal targets (around $5.0 \mu \mathrm{m}[20]$ ) and the plume expansion. The interaction between the atoms of the system was governed by the modified embedded atom method (MEAM) potential [27], which has been widely used in the MD simulation for face centered cubic (fcc) metal targets, such as aluminum, copper, silver, etc. In the MEAM formulation, the total energy $\mathrm{E}$ of a system of atoms is given by [27]

$$
E=\sum_{i}\left\{F_{i}\left(\rho_{i}\right)+\frac{1}{2} \sum_{i \neq j} \emptyset_{i j}\left(r_{i j}\right)\right\}
$$

where $F$ is the embedding energy which is a function of the atom electron density $\rho$, and $\phi$ is a pair potential interaction. The pair interaction is summed over all neighbors $j$ of atom $i$ within the cutoff distance. MEAM potential is applied in this calculation and the parameters for both aluminum and copper are taken from Ref. [27].

The instability of the liquid between the binodal and spinodal line is considered by addressing the density/thermal fluctuationsfor the atoms in the surface region [28]. If the liquid is in the superheated state (between the binodal and spinodal) and close to the binodal line, the density fluctuation could push the liquid phase to the spinodal line or pull back to the binodal line. With the increase of superheating, the free energy barrier separating the liquid and vapor states becomes lower. Under this condition, the thermal fluctuation could easily cross the barrier and facilitate the phase separation. This is further analyzed in the thermodynamic trajectory later in the results section. 
Fig. 5 shows the atom distribution at different time for the laser fluence of 12 $\mathrm{J} / \mathrm{cm}^{2}$. The initial solid target is located at the bottom half $(\mathrm{z}<=15 \mu \mathrm{m})$ of the calculation domain. With the laser energy coming from the top, the surface region is melted and the atoms begin to move upwards. Also some large clusters of atoms are formed in the region of 8 to $16 \mu \mathrm{m}$. Fig. 6 shows the atom distribution prediction at $t=60 \mathrm{~ns}$ from MD simulation under different laser fluences. Clearly with different laser energy input, different numbers of clusters are formed with different size.

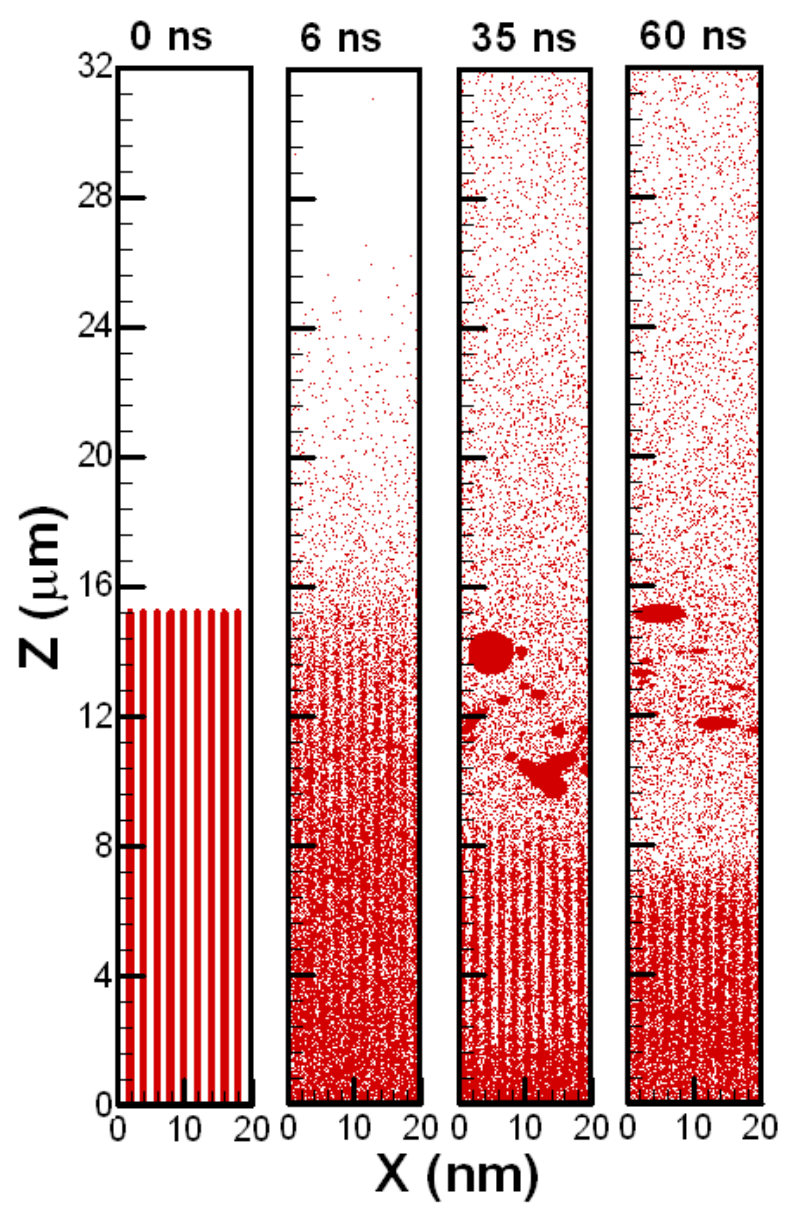

Fig. 5Atom distribution at different time (laser fluence $12 \mathrm{~J} / \mathrm{cm}^{2}$, wavelength $1064 \mathrm{~nm}$, pulse duration $6 \mathrm{~ns}$ ) 

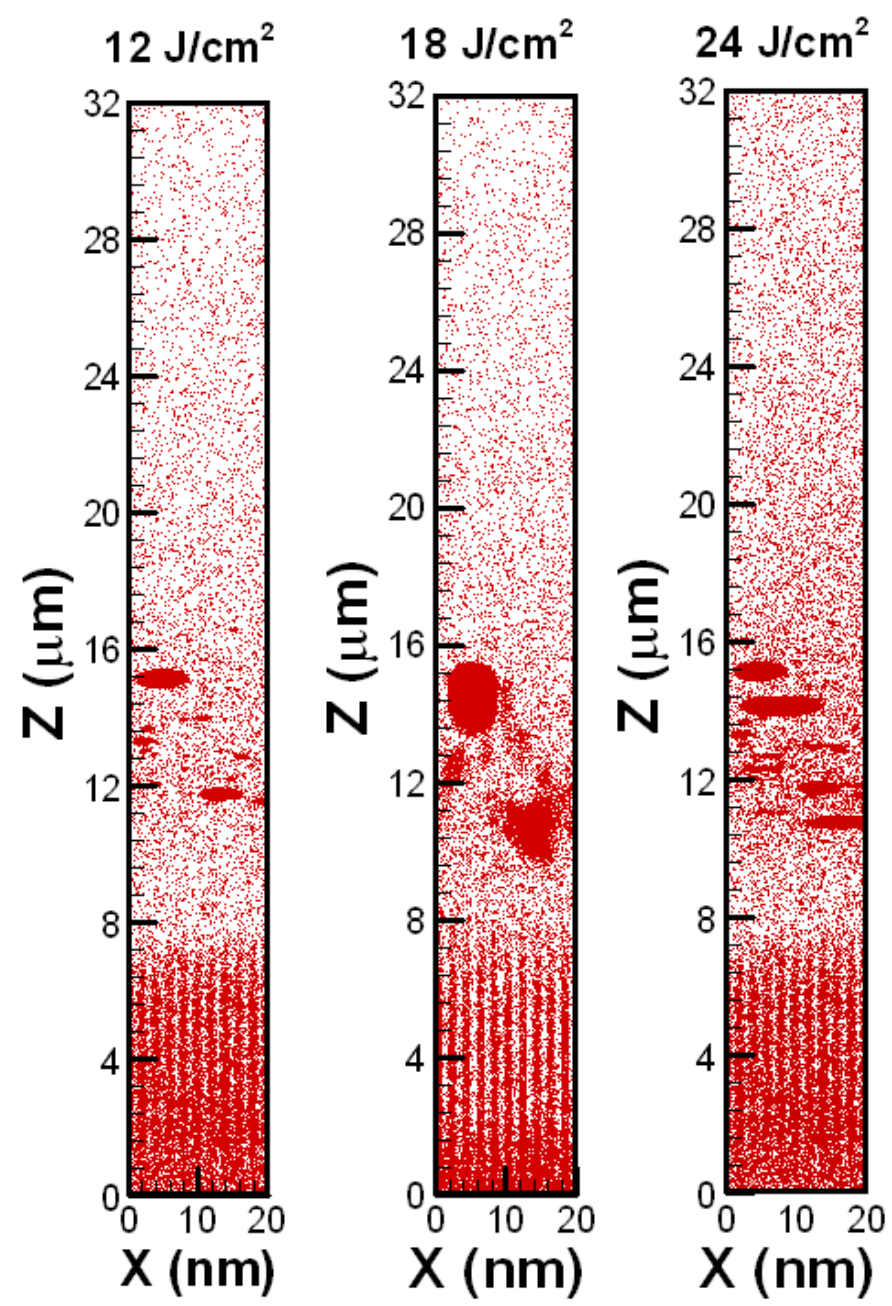

Fig. 6Atom distribution at $\mathrm{t}=60 \mathrm{~ns}$ from $\mathrm{MD}$ simulation under different laser fluences

The initial atom distributionsobtained from MD simulation can be combined according to the beam profile to get an atom distribution in a larger domain size using the periodic boundary assumption. By combining the MD simulation results, one can obtain the initial SPH particles distribution, as shown in Fig. 7. In this step, the initial SPH particles are assumed to be in the spherical shape. The location of the SPH particles in each large cell is determined by the initial atom positions and the mass distribution in the large cell. The temperature and velocity information of all the atoms can also be transferred to the corresponding SPH particles using the same method. 


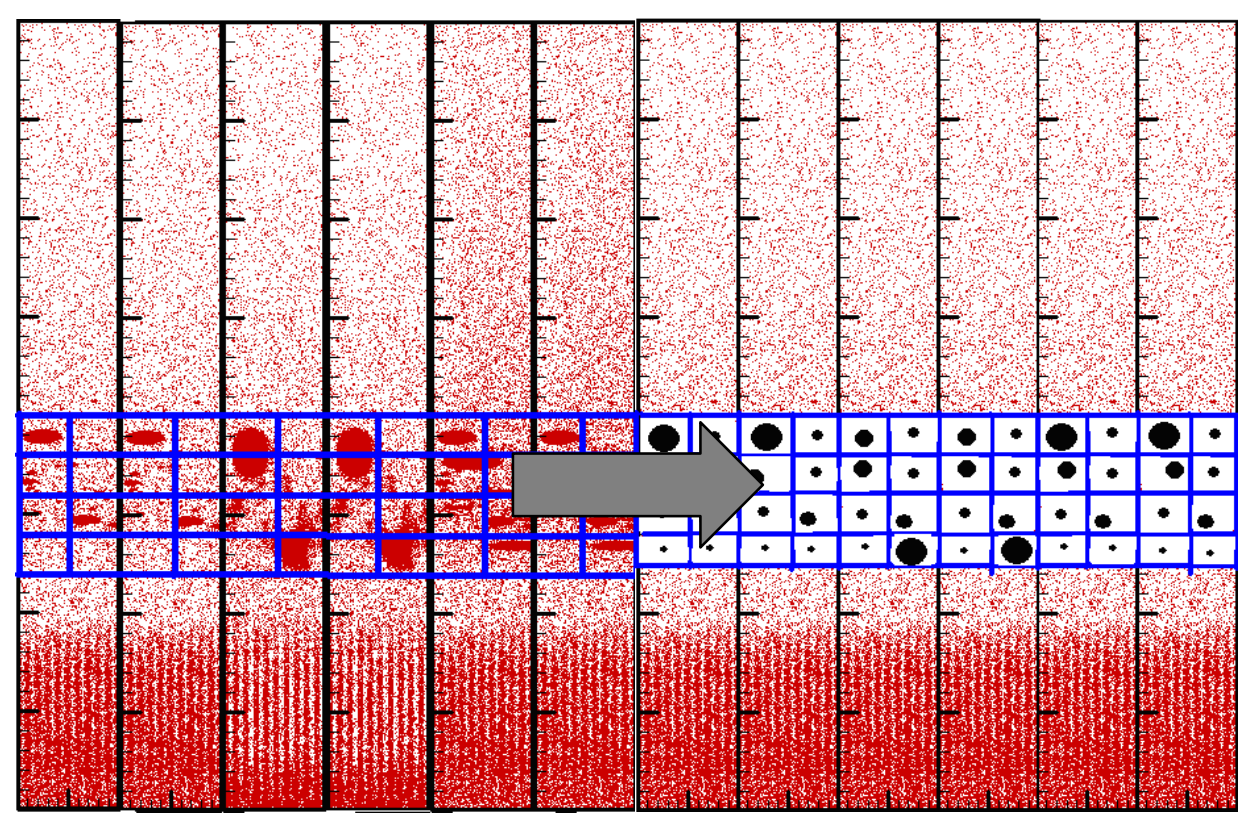

Fig. 7Mapping of particles predicted by MD to SPH particles

The SPH calculation then starts with the input from MD model. Fig. 8 shows the initial configuration of SPH calculation. The close-up view shows the particle distribution from the MD calculation, where particles are formed with different sizes. The bottom of the whole calculation domain is set to be stationary in the SPH calculation. With the above procedure, the SPH model should be able to calculate the temperature evolution inside the molten pool,the large particle movement, and eventually the melt ejection. 


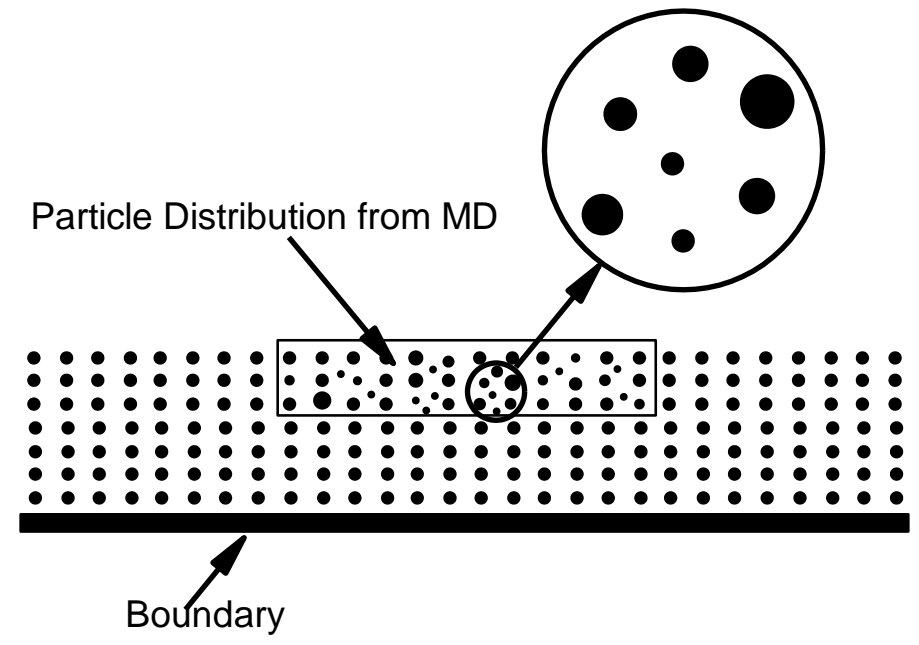

Fig. 8 Initial configuration of SPH calculation

\section{Experimental Setup and Procedures}

A probe beam-pump beam technique is used in this work to observe the phase explosion. Fig. 9 shows the experimental setup. The pump beam is produced by the NdYAG nanosecond laser (Continuum Surelite) operating at $1064 \mathrm{~nm}$ with a pulse duration of 6 ns. The Nd-YVO 4 picosecond laser (Lumera Rapid) is used to provide the green probe beam (532 nm, a pulse duration of $10 \mathrm{ps).} \mathrm{Two} \mathrm{photo} \mathrm{detectors} \mathrm{(with} \mathrm{photodiode}$ inside, ThorLabs, DET200) are used in the experimental setup to synchronize the probe beam (ps laser) and pump beam (ns laser). To control the exposure time accurately and obtain the images at different time, the CCD camera (Imaging Source, DFK 42BUC03) needs to be externally triggered and synchronized to work with the probe beam/pump beam together. 


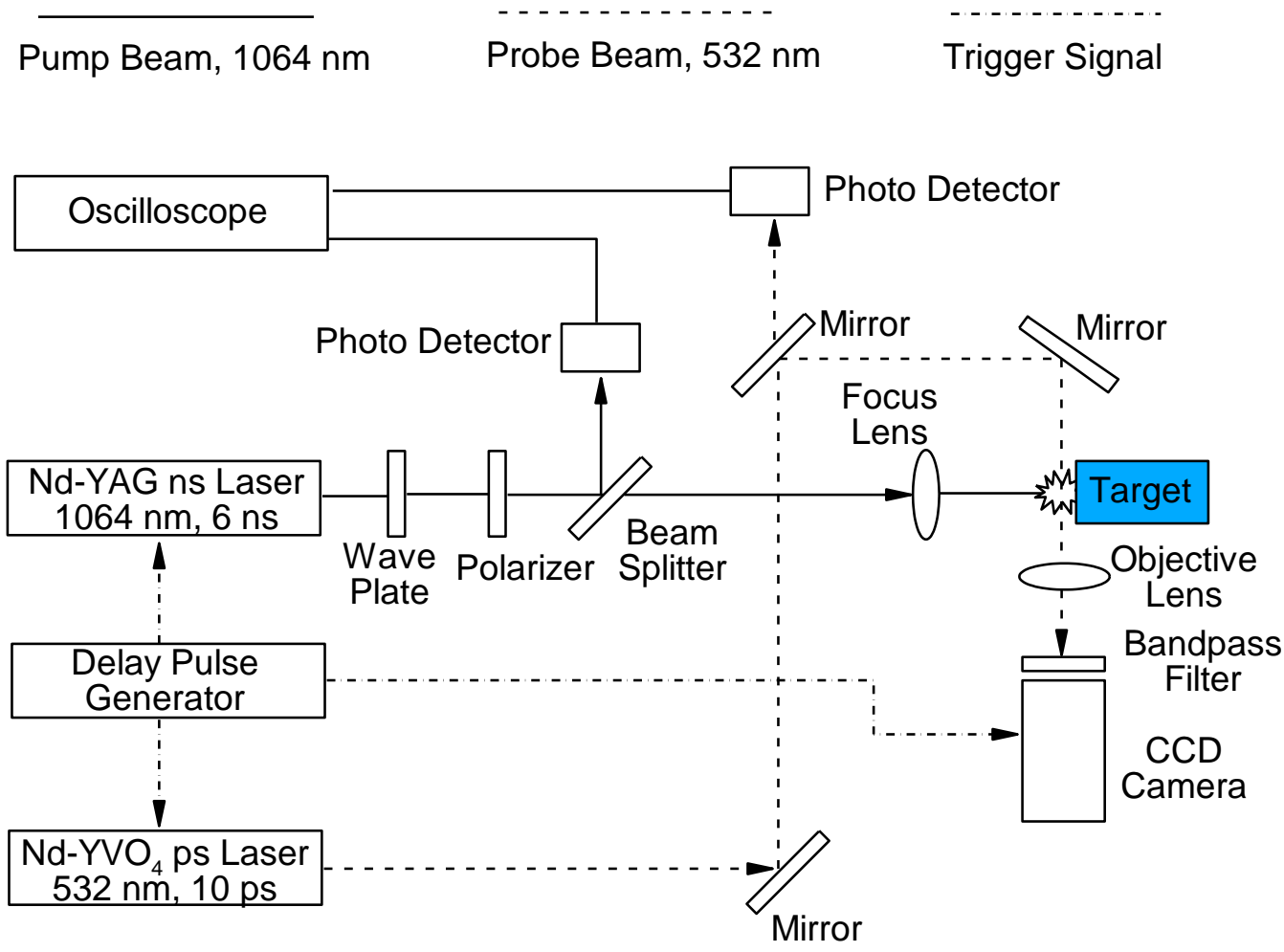

Fig. 9 Schematic drawing of the experimental setup

The probe beam laser, the pump beam laser, and the CCD camera are triggered externally by the delay pulse generator (BNC 555). The time sequence of the triggering signals is shown in Fig. 10. For the ps laser, the $1120 \mathrm{~ns}$ is the default delay time before a laser pulse could be generated when a trigger signal is sent to the laser. Similarly, $137 \mu$ s and $240.1 \mu$ s are the default delay time for the CCD camera and the ns laser, respectively. To precisely determine when the CCD images are taken, the ps laser and ns are synchronized first and then the CCD camera is added later. It should be noted that the delay time between the trigger signals are carefully controlled to make sure that the probe ps beam illuminates the ns laser ablation site during the CCD exposure period. To eliminate the effect of background light, the experiments are conducted in a dark 
environment. By changing the delay time between the nanosecond laser pulse and the picosecond laser, one can capture the images at different time instants after the nanosecond laser pulse irradiates the target surface.

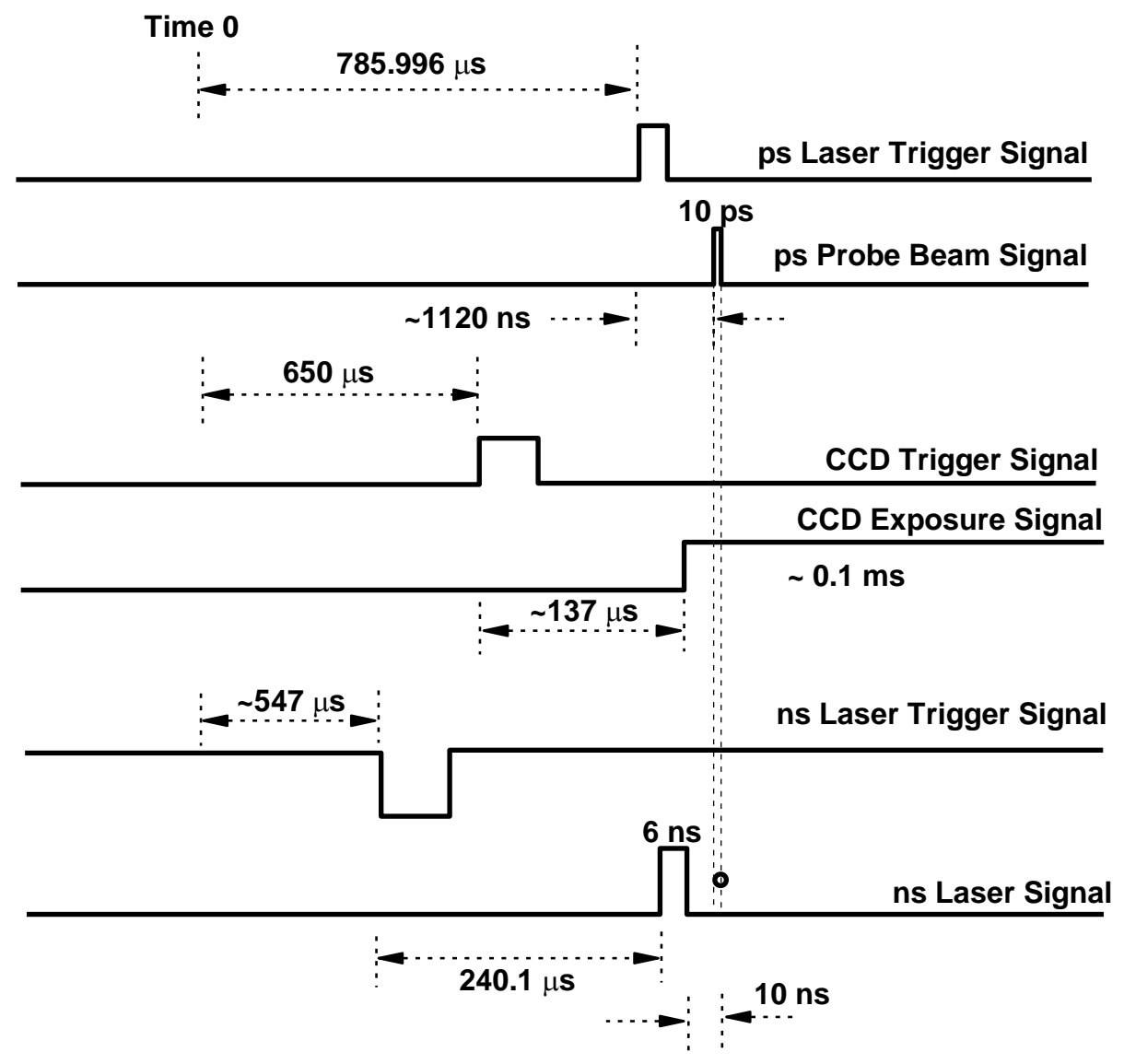

Fig. 10 Time sequence of triggering signals

\section{Results and Discussion}

\subsection{Laser Ablation of Aluminum}

To compare the experiment results with the simulation data, the MD/SPH simulation of the laser ablation process was conducted under the same condition. The melt ejection 
could be calculated using the SPH model with the initial particle distribution from MD calculation.

Case A: Laser Fluence at $12 \mathrm{~J} / \mathrm{cm}^{2}$

The laser fluence in this case is right above the phase explosion threshold according to the previous HD calculation [20]. Fig. 11 shows the calculation results at different time instants for this case. In all the CCD images and prediction results shown in this work, time 0 is defined as the instant when the ns laser beam irradiates the target surface. Some large particles can be observed to being ejected from the melt pool at $72 \mathrm{~ns}$, as seen in Fig. 11 (b). Therefore, the starting time of melt ejection (phase explosion) is around $72 \mathrm{~ns}$ after the ns laser beam irradiates the target surface. The experimental results from Poneala and Willis [8] indicated a starting time between 52 and 114 ns under a similar condition.

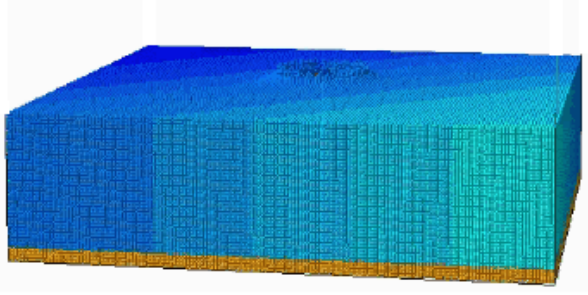

(a) $66 \mathrm{~ns}$

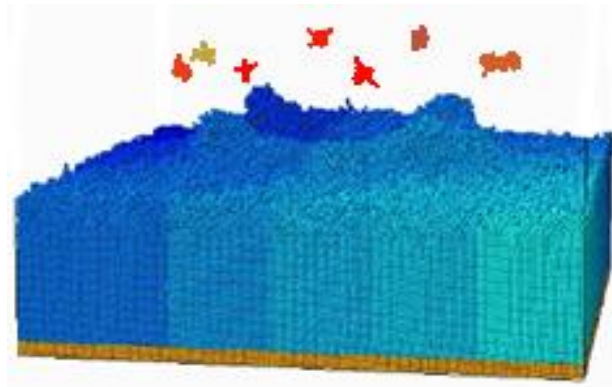

(c) $78 \mathrm{~ns}$

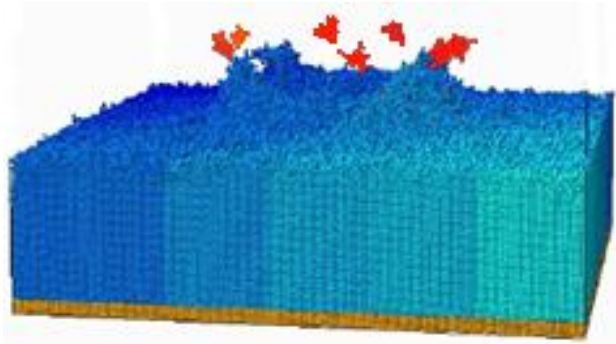

(b) $72 \mathrm{~ns}$

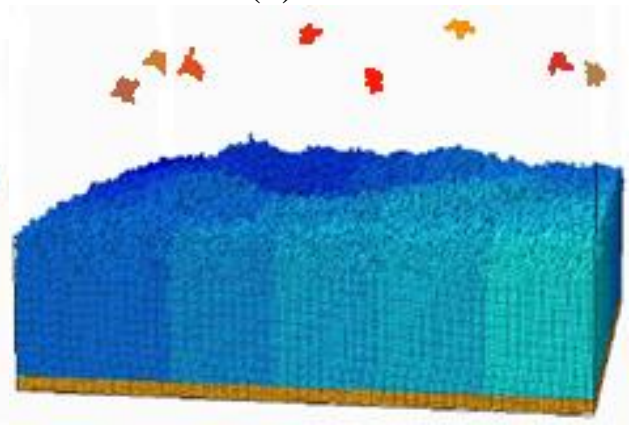

(d) $84 \mathrm{~ns}$

Fig. 11 Modeling results of melt ejection after laser ablation (laser fluence $12 \mathrm{~J} / \mathrm{cm}^{2}$, pulse duration $6 \mathrm{~ns}, 1064 \mathrm{~nm}$, beam diameter $200 \mu \mathrm{m}$ ) 
Fig. 12 shows the experimental observation under the same condition. In the CCD images shown, the bottom boundary is the target surface. The center region is the ns laser ablation site. The laser beam irradiates the target surface from the top of the image. At around $\mathrm{t}=70 \mathrm{~ns}$, some particles (black dots) can be observed in the bottom-center region. With the increase of time, more particles are ejected from the melt pool. The particles move vertically first and then expands in the radial direction, which is very similar to the model prediction shown in Fig. 11. Further analysis about the particle size distribution at different time will be shown later.

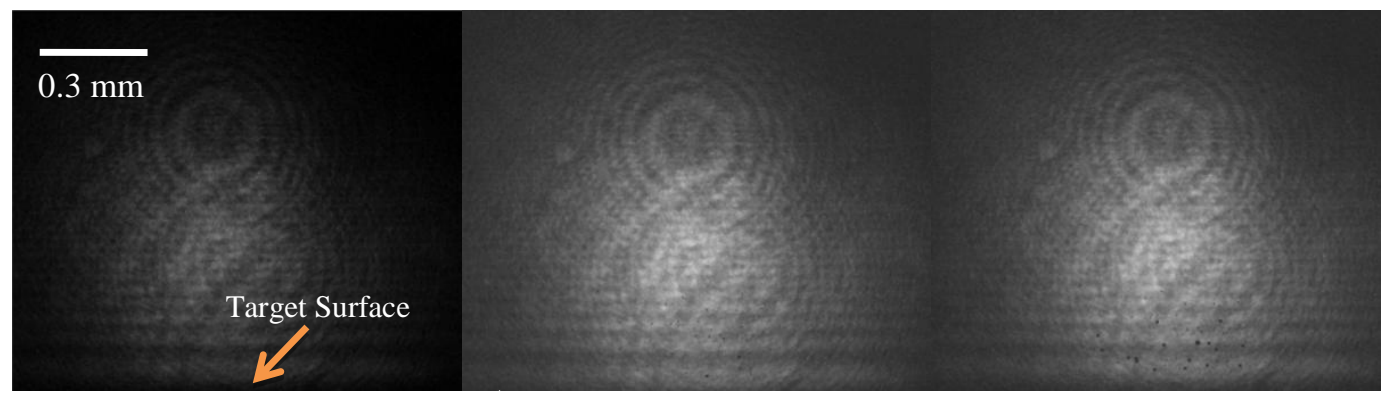
(a) $\mathrm{t}=60 \mathrm{~ns}$
(b) $\mathrm{t}=65 \mathrm{~ns}$
(c) $\mathrm{t}=70 \mathrm{~ns}$

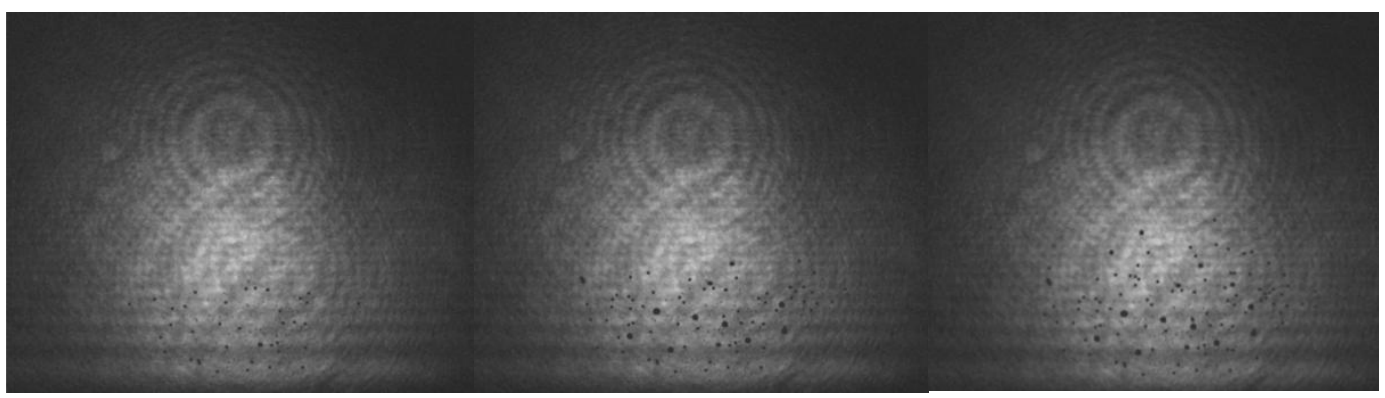
(d) $\mathrm{t}=75 \mathrm{~ns}$
(e) $\mathrm{t}=80 \mathrm{~ns}$
(f) $\mathrm{t}=85 \mathrm{~ns}$

Fig. 12 Experimental observation of melt ejection under laser fluence $12 \mathrm{~J} / \mathrm{cm}^{2}$ (laser beam coming from the top of the image, pulse duration $6 \mathrm{~ns}, 1064 \mathrm{~nm}$, beam diameter $200 \mu \mathrm{m})$ 
Case B: Laser Fluence at $24 \mathrm{~J} / \mathrm{cm}^{2}$

In this test case, the laser fluence is $24 \mathrm{~J} / \mathrm{cm}^{2}$. Phase explosion will occur in this case. Fig. 13 shows the calculation results of the melt ejection from $t=66$ ns to $t=84$ ns. Fig. 14 shows the experimental observation under the same condition.

Similar to the previous case, the particle ejection starts at around $t=70 \mathrm{~ns}$ in both the model prediction and experimental observation. Due to the higher laser fluence than that in Case A, stronger ejection can be observed at the later stage in this case. Both the model prediction and the experimental observation indicate that the particles move up first and then expand to the radial direction later. Also, the model prediction shows that more particles are ejected from the melt pool than that in the experimental observation, especially in the later stage. This observation will be further analyzed in the next section.

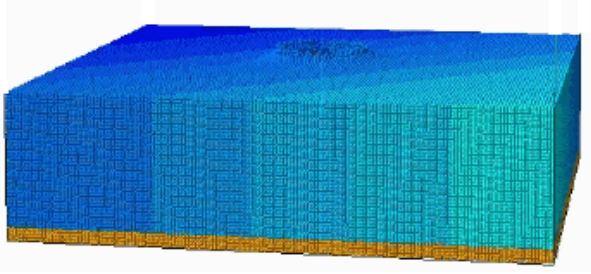

(a) $66 \mathrm{~ns}$

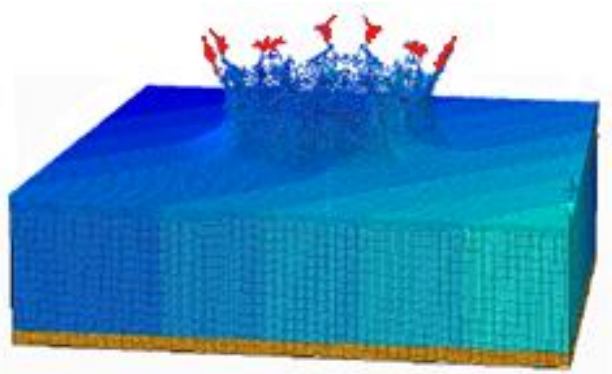

(c) $78 \mathrm{~ns}$

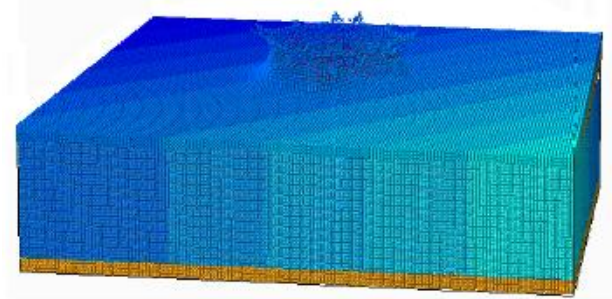

(b) $72 \mathrm{~ns}$

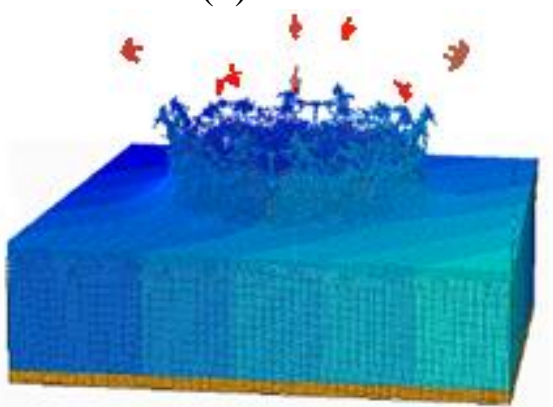

(d) $84 \mathrm{~ns}$

Fig. 13 Modeling results of melt ejection after laser ablation (laser fluence $24 \mathrm{~J} / \mathrm{cm}^{2}$, pulse duration $6 \mathrm{~ns}, 1064 \mathrm{~nm}$, beam diameter $200 \mu \mathrm{m}$ ) 


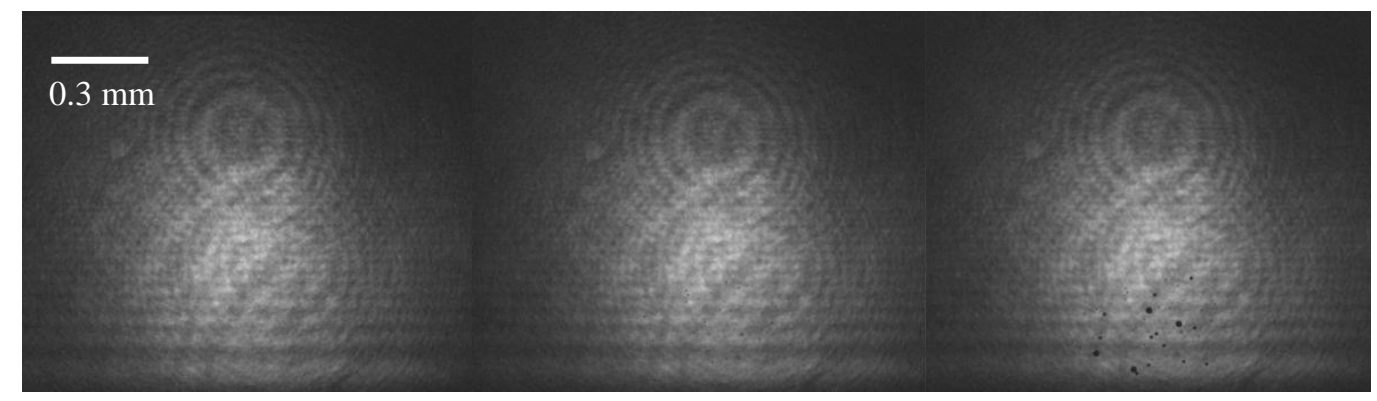
(a) $\mathrm{t}=60 \mathrm{~ns}$
(b) $t=65 n s$
(c) $\mathrm{t}=70 \mathrm{~ns}$

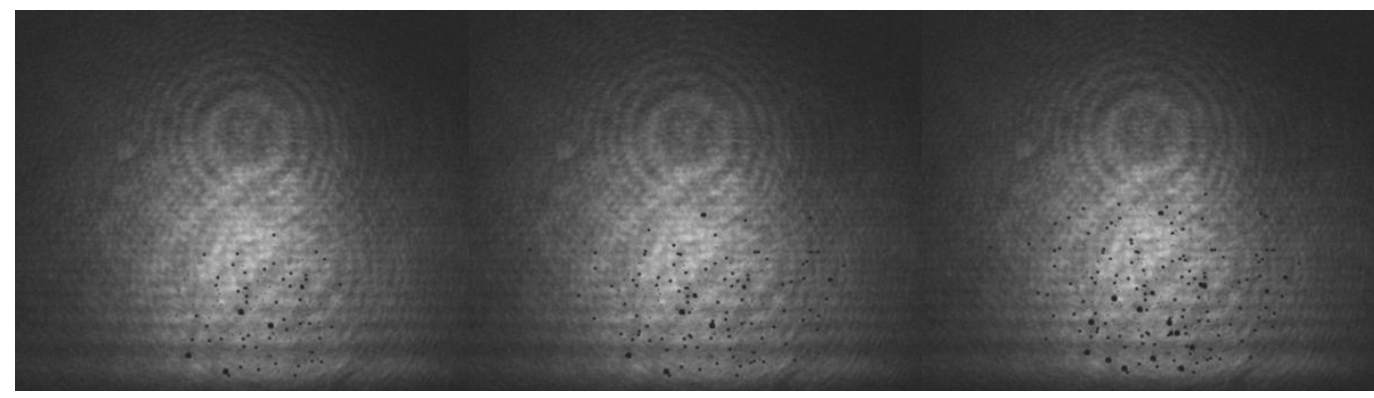
(d) $\mathrm{t}=75 \mathrm{~ns}$
(e) $\mathrm{t}=80 \mathrm{~ns}$
(f) $\mathrm{t}=85 \mathrm{~ns}$

Fig. 14 Experimental observation of melt ejection under laser fluence $24 \mathrm{~J} / \mathrm{cm}^{2}$ (laser beam coming from the top of the image,pulse duration $6 \mathrm{~ns}, 1064 \mathrm{~nm}$, beam diameter 200 $\mu \mathrm{m})$

\section{Case C: Laser Fluence at $36 \mathrm{~J} / \mathrm{cm}^{2}$}

Fig. 15 shows the melt ejection predicted by the SPH calculation under laser fluence of $36 \mathrm{~J} / \mathrm{cm}^{2}$. Fig. 16 shows the experimental observation under the same condition. With the highest laser fluence, much stronger particle ejection can be observed in this case. The average particle size is also larger than the previous two cases. The ejection starting time is a little earlier in this case, which is around $t=65 \mathrm{~ns}$. The ejected particles move up first in the vertical direction and then expand to the radial direction at the later stage. 


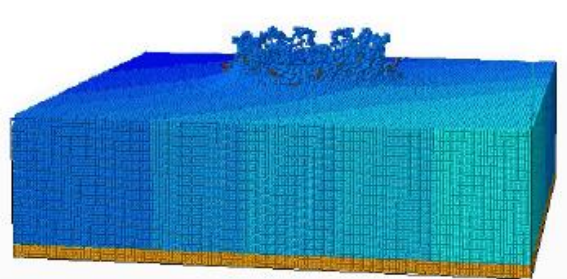

(a) $66 \mathrm{~ns}$

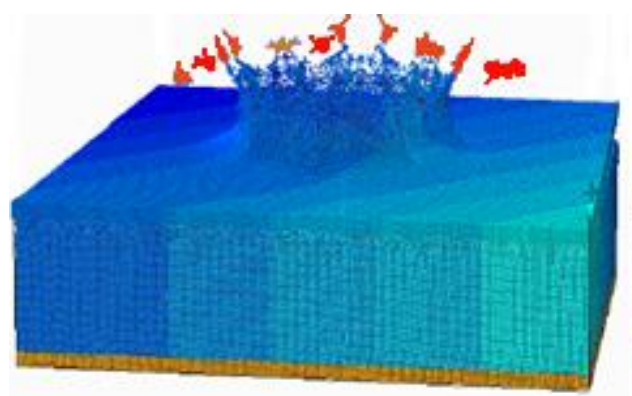

(c) $78 \mathrm{~ns}$

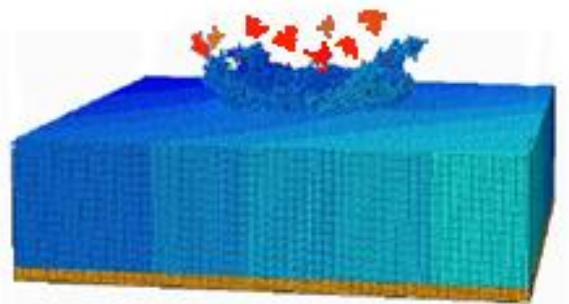

(b) $72 \mathrm{~ns}$

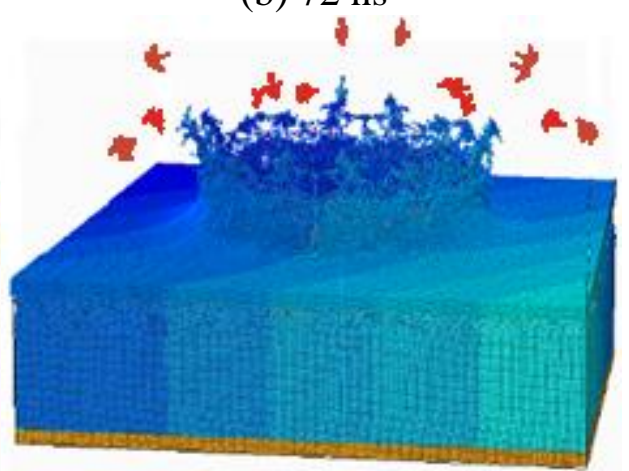

(d) $84 \mathrm{~ns}$

Fig. 15 Modeling results of melt ejection after laser ablation (laser fluence $36 \mathrm{~J} / \mathrm{cm}^{2}$, pulse duration $6 \mathrm{~ns}, 1064 \mathrm{~nm}$, beam diameter $200 \mu \mathrm{m}$ )

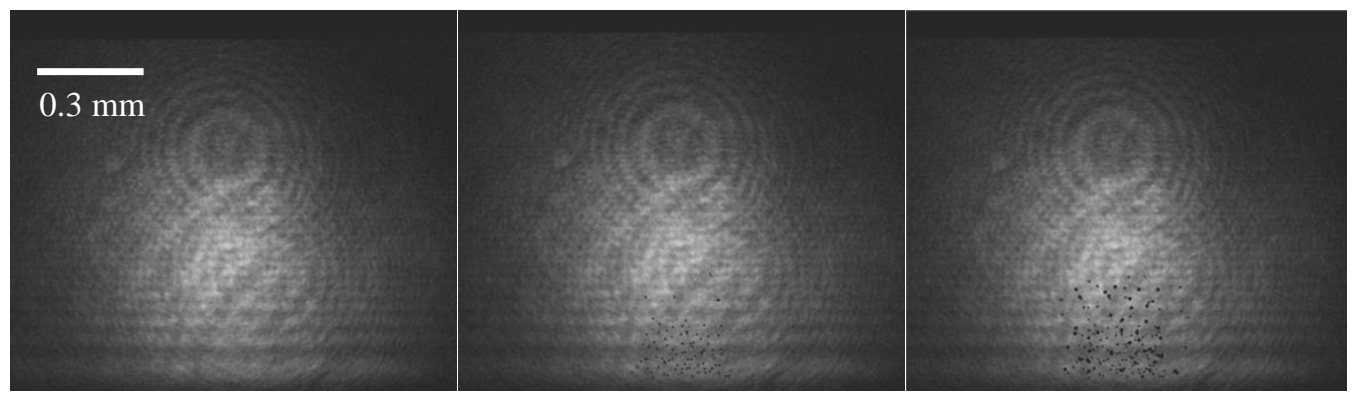
(a) $\mathrm{t}=60 \mathrm{~ns}$
(b) $\mathrm{t}=65 \mathrm{~ns}$
(c) $\mathrm{t}=70 \mathrm{~ns}$
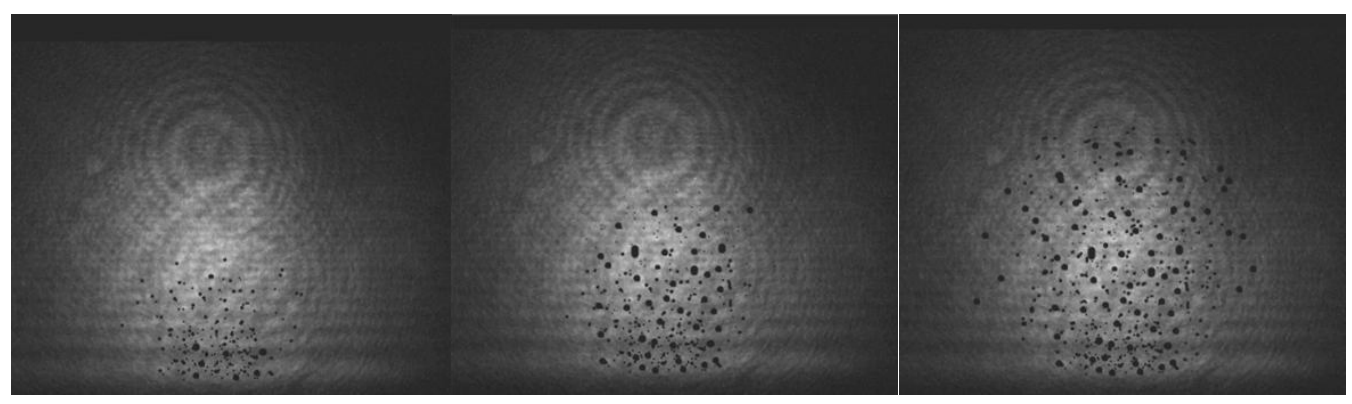

(d) $\mathrm{t}=75 \mathrm{~ns}$

(e) $\mathrm{t}=80 \mathrm{~ns}$

(f) $t=85 n s$

Fig. 16 Melt ejection under laser fluence $36 \mathrm{~J} / \mathrm{cm}^{2}$ (laser beam coming from the top of the image,pulse duration $6 \mathrm{~ns}, 1064 \mathrm{~nm}$, beam diameter $200 \mu \mathrm{m}$ ) 
In all three cases, the particle movement can be clearly seen in the figures, expanding vertically first and then radially in the later stage in both simulation and experimental observation. With the increase of the laser fluence, more particles could be observed with the CCD camera and also in the simulation. The average particle size tends to be larger in the case of higher laser fluence as well, due to the stronger laser-matter interaction at the high fluence.

To quantitatively compare the simulation results with the experimental observation, the particle size distributions are extracted from both figures under the laser fluence of 36 $\mathrm{J} / \mathrm{cm}^{2}$. Fig. 17 shows the comparison of particle size distributions at different time in a three-dimensional waterfall plot. It can be seen in Fig. 17 that both the distributions show a bi-modal shape, especially at the later stage. Also with the increase of the time, the particle size tends to increase in both plots. Overall the two distributions are very close. 


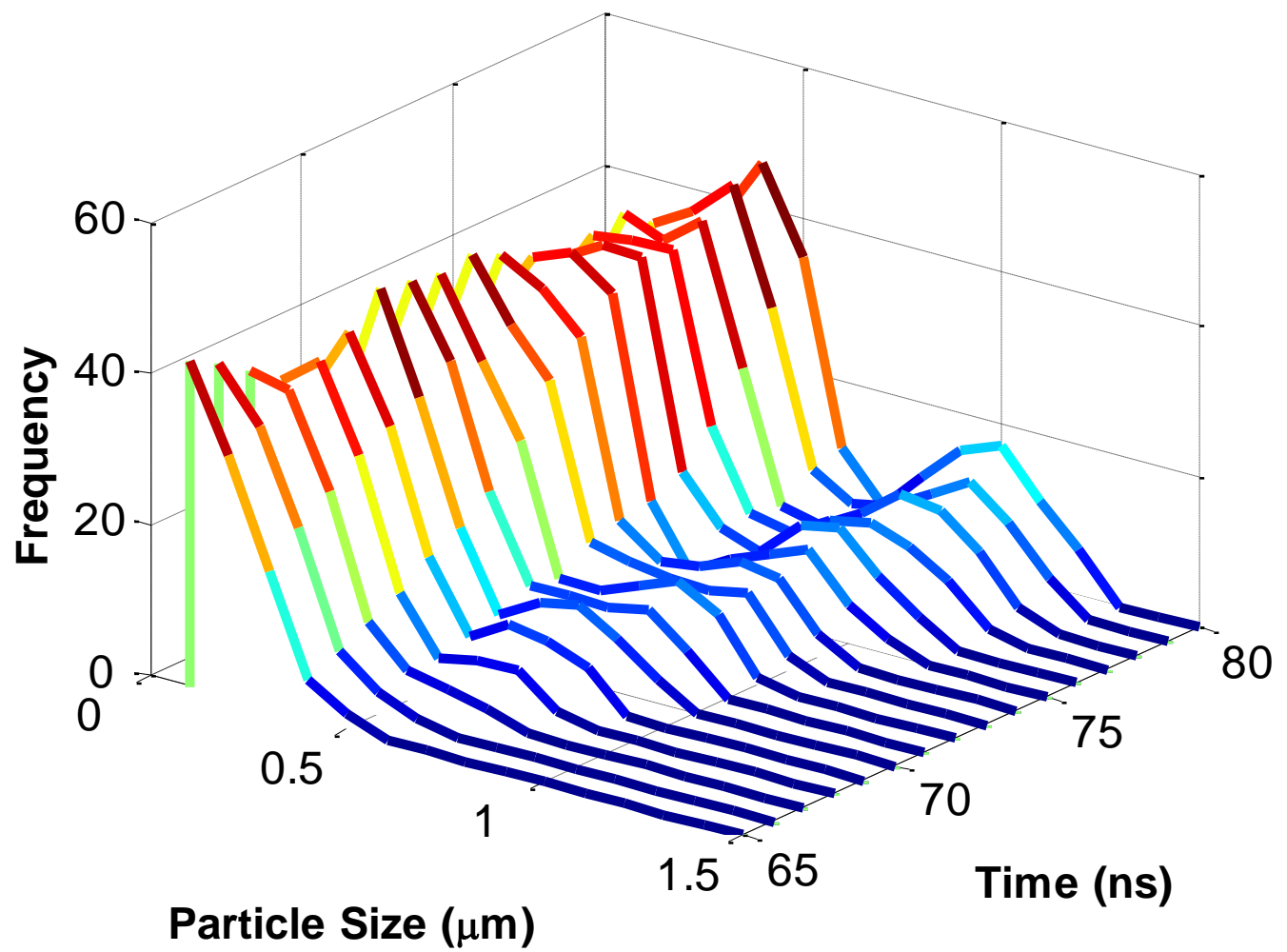

(a)

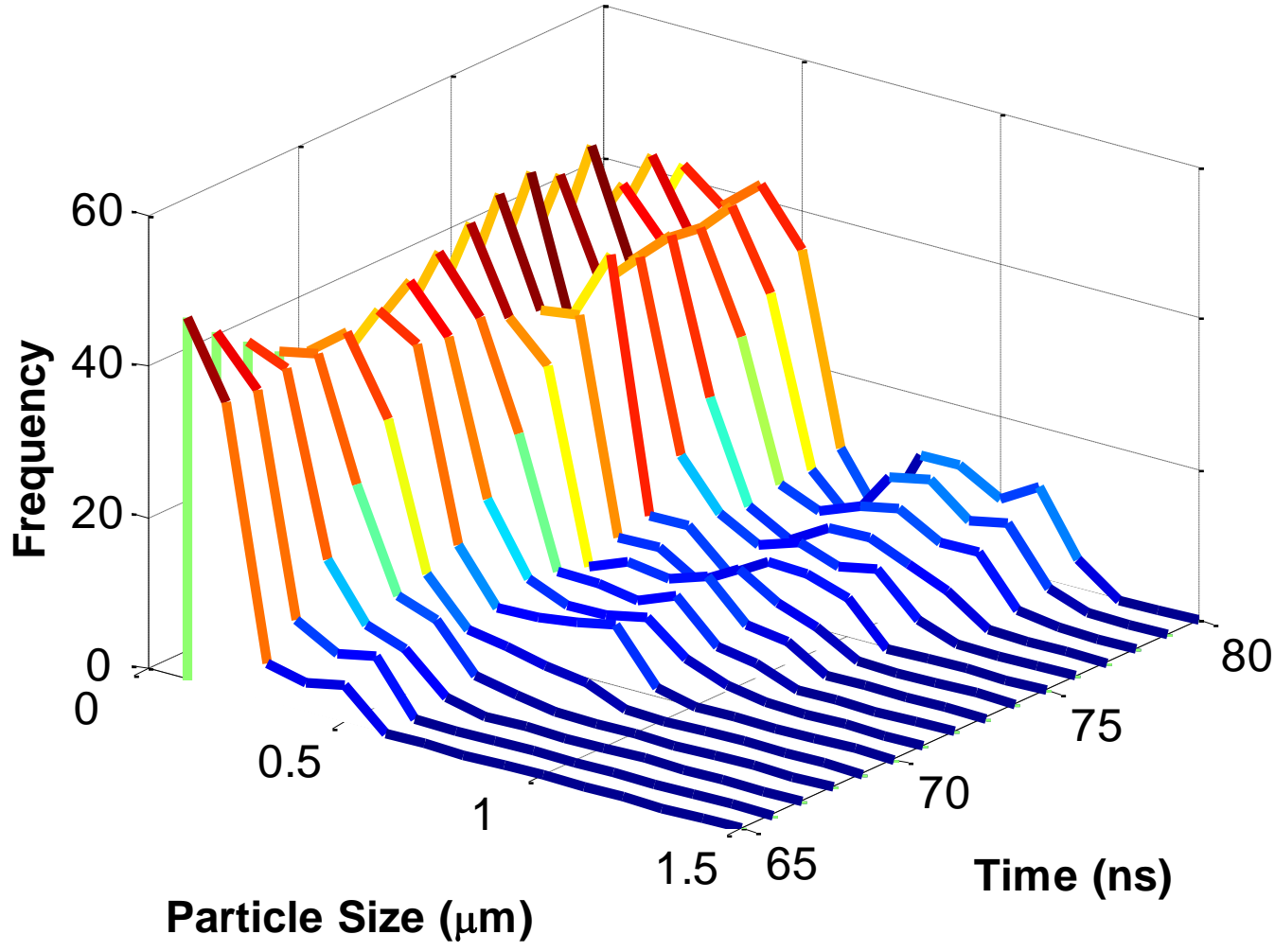

(b)

Fig. 17 Particle distribution from (a) simulation and (b) experiment (laser fluence 36 $\mathrm{J} / \mathrm{cm}^{2}$, pulse duration $6 \mathrm{~ns}, 1064 \mathrm{~nm}$, beam diameter $200 \mu \mathrm{m}$ ) 
To further understand the mechanism of the phase explosion, the temperature distribution inside the melt pool at different time is carefully investigated. Fig. 18 shows the initial temperature and subsequent temperature distributions inside the aluminum target at different time instants. The high temperature region inside the melt pool generally propagates into the deeper region with time. The temperature information at 65 ns indicates that the temperature at the bottom of the crater (around $-4 \mu \mathrm{m}$ ) is around $5500 \mathrm{~K}$, which is close to the $0.9 \mathrm{~T}_{\mathrm{c}}$ value for aluminum (around $5600 \mathrm{~K}$ ). The prediction of the ablation depth from the HD model using the $0.9 \mathrm{~T}_{\mathrm{c}}$ criterion is also around $4 \mu \mathrm{m}$. This confirms that the $0.9 \mathrm{~T}_{\mathrm{c}}$ criterion is reasonable for predicting ablation depth with the occurrence of phase explosion in the HD calculation for aluminum.

Since the critical temperature is very important in the current work, its value is further analyzed with MD simulation following the method developed in Ref. [29]. By calculating multiple isotherms near the critical point, as seen in Fig. 19, the critical temperature is predicted to be around $5950 \pm 20 \mathrm{~K}$, which is close to the value $(6200 \mathrm{~K})$ used here for aluminum. The literature reported value for the critical temperature of aluminum is in the range of 5400-9500 K [30]. However, recent estimates of the value are in the low end of the range, for example, $6700 \pm 800 \mathrm{~K}$ in [30], $6300 \mathrm{~K}$ in [31]. Therefore the value used in this work $(6200 \mathrm{~K})$ is in the reasonable range. 


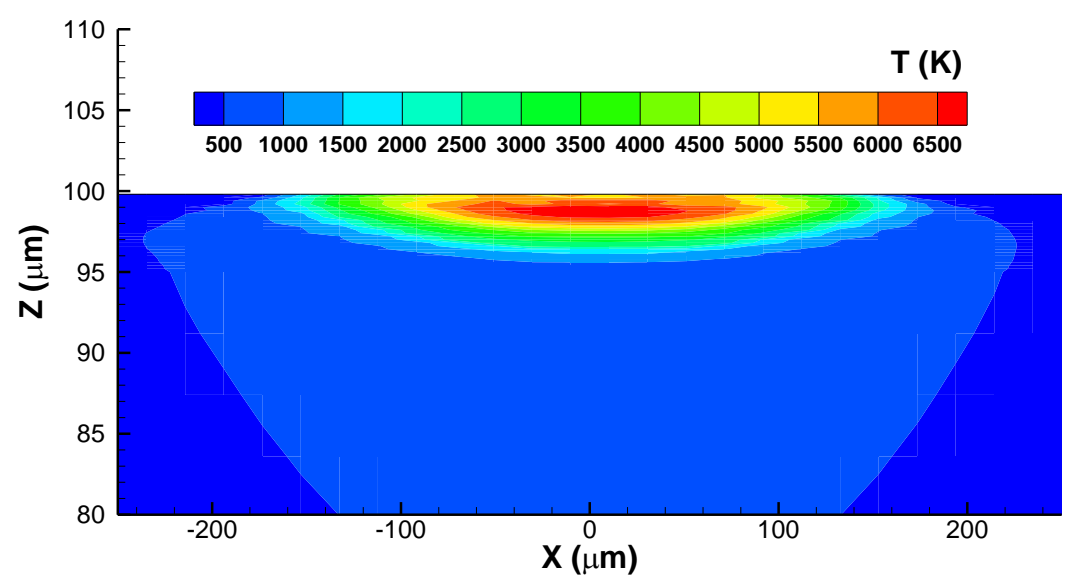

(a) Initial temperature distibution at $\mathrm{t}=35 \mathrm{~ns}$ for $\mathrm{SPH}$ calcualtion

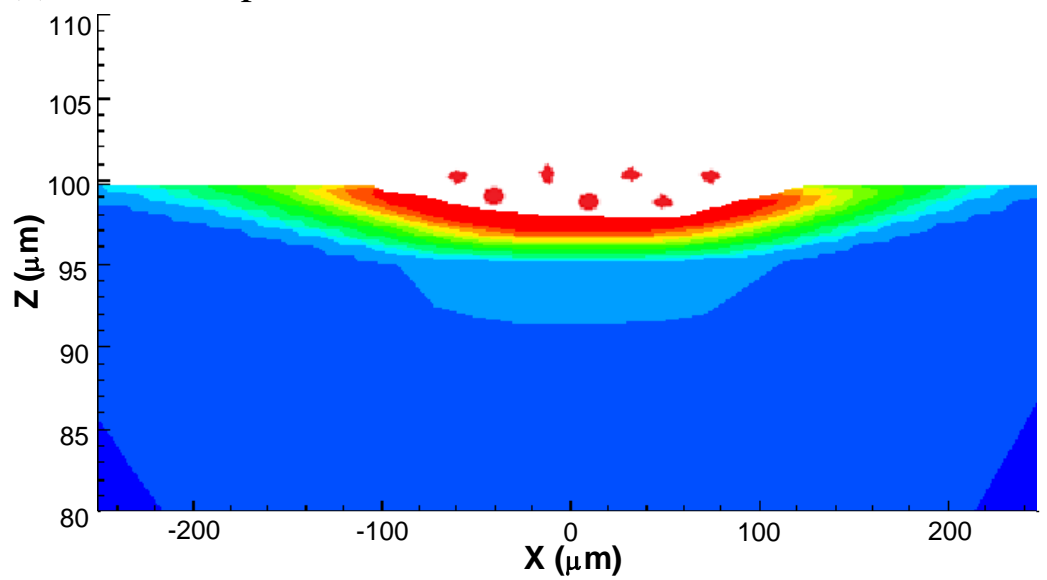

(b) $\mathrm{t}=50 \mathrm{~ns}$

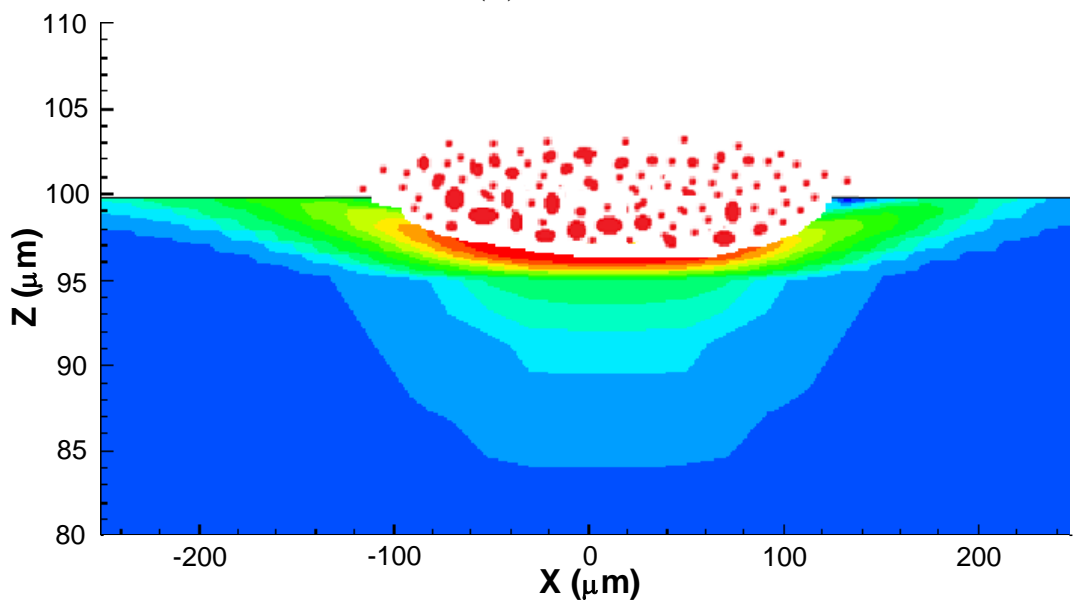

(c) $\mathrm{t}=65 \mathrm{~ns}$

Fig. 18 Temperature distribution inside the aluminum target at different time (laser fluence $36 \mathrm{~J} / \mathrm{cm}^{2}$, pulse duration $6 \mathrm{~ns}, 1064 \mathrm{~nm}$, beam diameter $200 \mu \mathrm{m}$ ) 


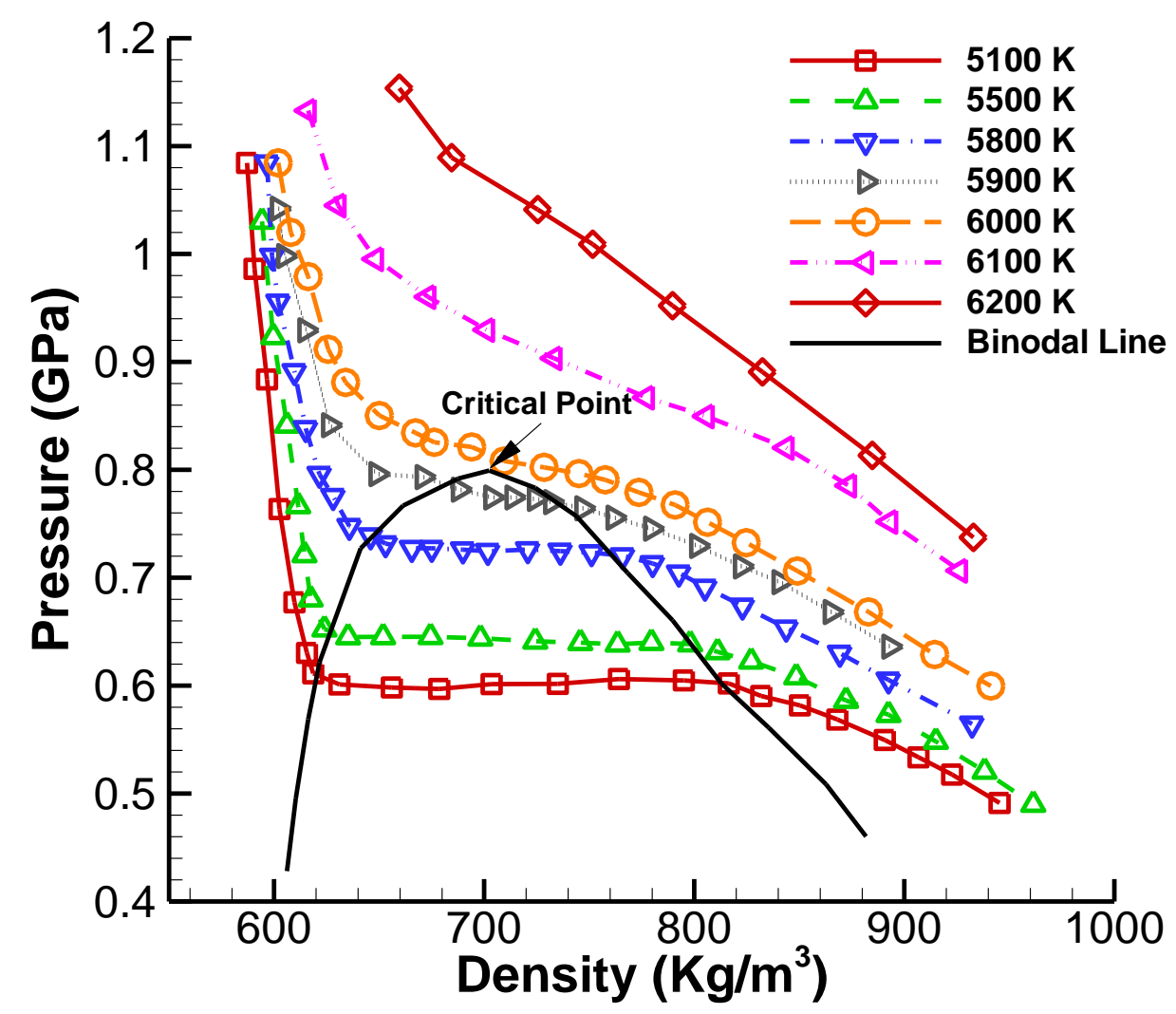

Fig. 19 Calculated isotherms from MD simulation for Aluminum

The thermodynamic trajectories of the different aluminum particles at different regions are further analyzed and shown in Fig. 20. In this figure, the spinodal and binodal curves are marked as well as the super-heated liquid (SHL) region. The numbers marked along the thermodynamic trajectory are the time instants (in ns) in the calculation. Clearly, the aluminum particles at 4.0 microns below the original surface will enter the unstable zone and go through the spinodal decomposition process [32]. As a result, these particles will be ejected from the melt pool, as observed in both the model prediction and experimental observation in Case $\mathrm{C}$. The particles in the deeper zone will solidify back to the bulk solid state as indicated by the thermodynamic trajectories in Fig. 20. 


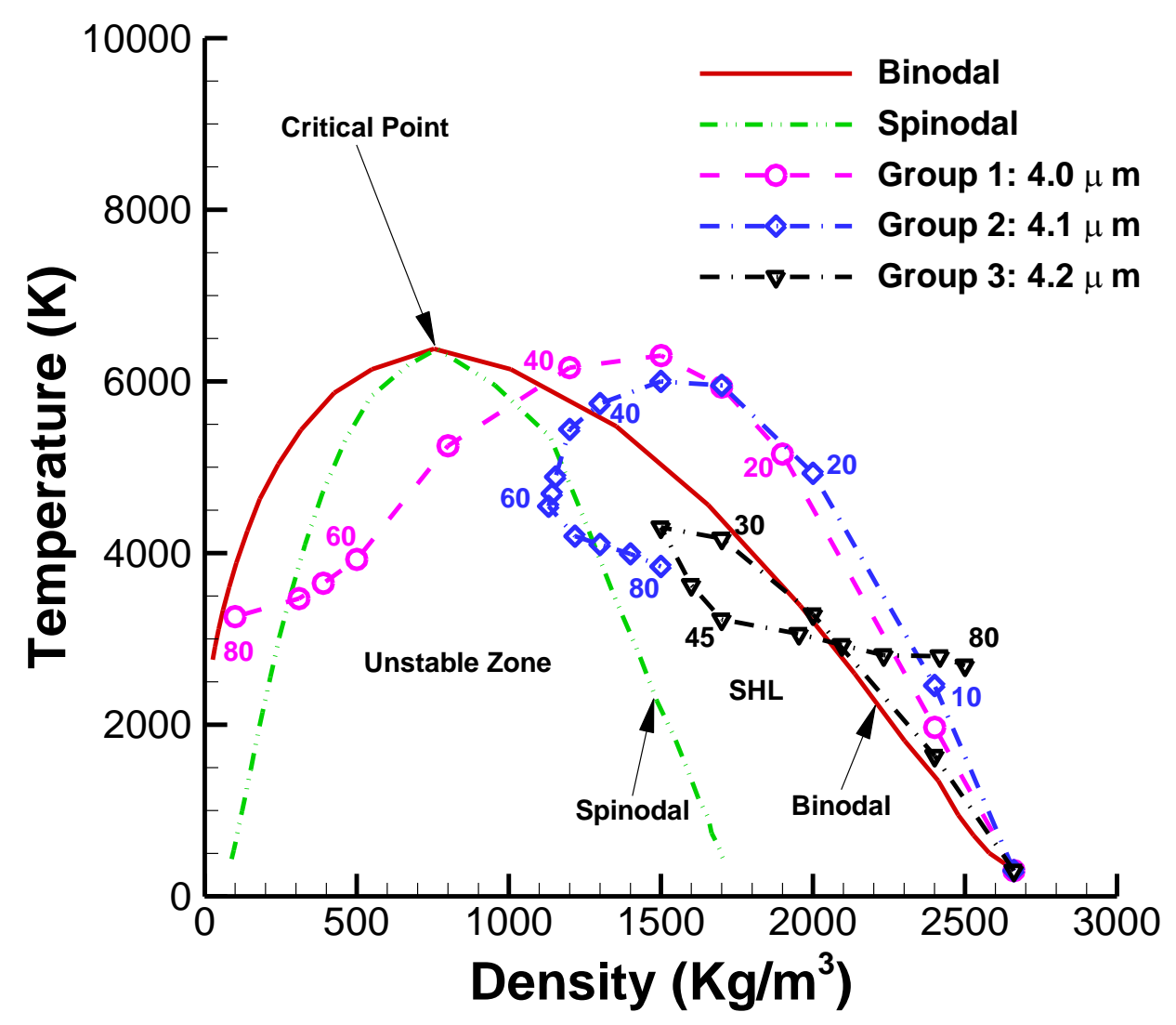

Fig. 20 Thermodynamic trajectory of the aluminum inside the melt pool (laser fluence 36 $\mathrm{J} / \mathrm{cm}^{2}$, pulse duration $6 \mathrm{~ns}, 1064 \mathrm{~nm}$, beam diameter $200 \mu \mathrm{m}$ )

\subsection{Laser Ablation of Copper}

To evaluate the validity of 0.9 Tc criterion during phase explosion, laser ablation of copper is also investigated numerically with the MD/SPH model under different laser fluences and experimentally with the experimental setup shown in Fig. 9. According to Ref. [33-34], phase explosion should occur when the laser fluence is greater than 30 $\mathrm{J} / \mathrm{cm}^{2}$ for copper for a nanosecond laser operating at $1064 \mathrm{~nm}$ and a pulse duration of 6 ns. In this experiment, the laser fluencesare chosen to be $36 \mathrm{~J} / \mathrm{cm}^{2}$ or higher to make sure that the phase explosion could be observed by the CCD camera. Fig. 21 shows the experimental observation at different delay time. 


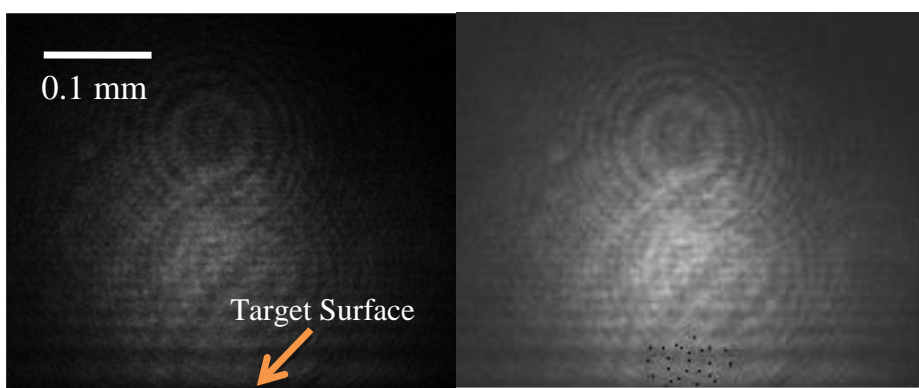

(a) t $=50 \mathrm{~ns}$

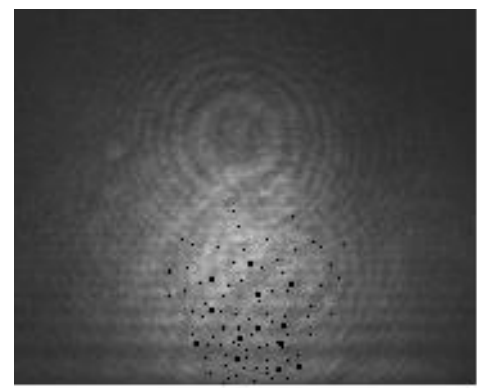

(d) $\mathrm{t}=65 \mathrm{~ns}$ (b) $\mathrm{t}=55 \mathrm{~ns}$

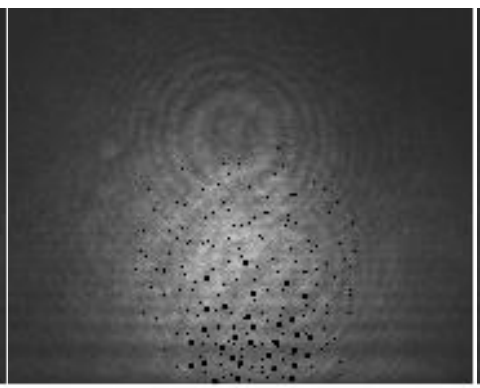

(e) $\mathrm{t}=70 \mathrm{~ns}$

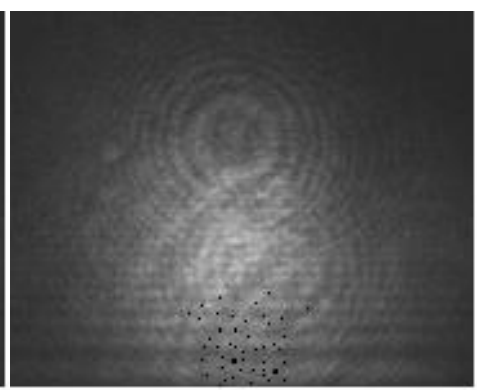

(c) $\mathrm{t}=60 \mathrm{~ns}$

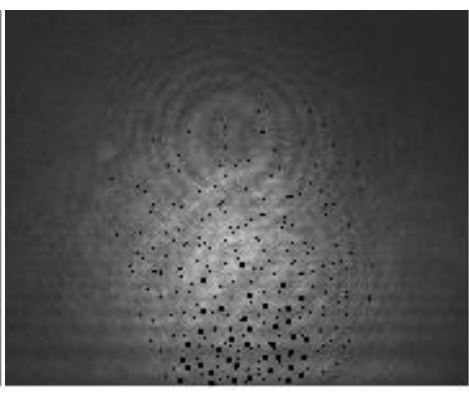

(f) $\mathrm{t}=75 \mathrm{~ns}$

Fig. 21 Experimental observation of melt ejection under laser fluence $36 \mathrm{~J} / \mathrm{cm}^{2}$ (copper target, laser beam coming from the top of the image, pulse duration $6 \mathrm{~ns}, 1064 \mathrm{~nm}$, beam diameter $100 \mu \mathrm{m})$

Similar to the case of aluminum, the melt ejection starts at around 50 to $55 \mathrm{~ns}$. The ejected particles move upwards first and then expand to the radial direction. With the increase of time, some larger particles can be observed in the CCD images, mostly residing in the region close to the target surface. The major difference between the aluminum and copper case is that a smallerbeam diameter $(100 \mu \mathrm{m}$ vs. $200 \mu \mathrm{m})$ wasused in the copper case. According to the experimental observation, the average particle size in the copper case is a bit smaller than that in the aluminum case. Based on the kinetic theory [16-18], phase explosion occurs when the vapor bubbles generated in the superheated liquid grow to a critical radius and expand spontaneously, which depends on the surface tension, critical temperature, pressure of superheated liquid, etc. The critical radius is estimated to be $0.5 \mu \mathrm{m}$ for copper and $0.7 \mu \mathrm{m}$ for aluminum. If one can assume 
that the ejected particle size is closely related to the critical radius of vapor bubble, this might explain the smaller particle observation seen in Fig.21. Considering a relatively larger amount of ejected particles in the same volume, one can expect a higher ablation depth in the copper case. The ablation profile is then measured with an optical 3D surface profilometer (KLA-Tencor, MicroXAM-100), as seen in Fig. 22. Based on the ablation profile, the ablation depth is estimated to be around $5.0 \mu \mathrm{m}$, which is indeed higher than that for aluminum $(4.0 \mu \mathrm{m})$ under the same laser fluence.

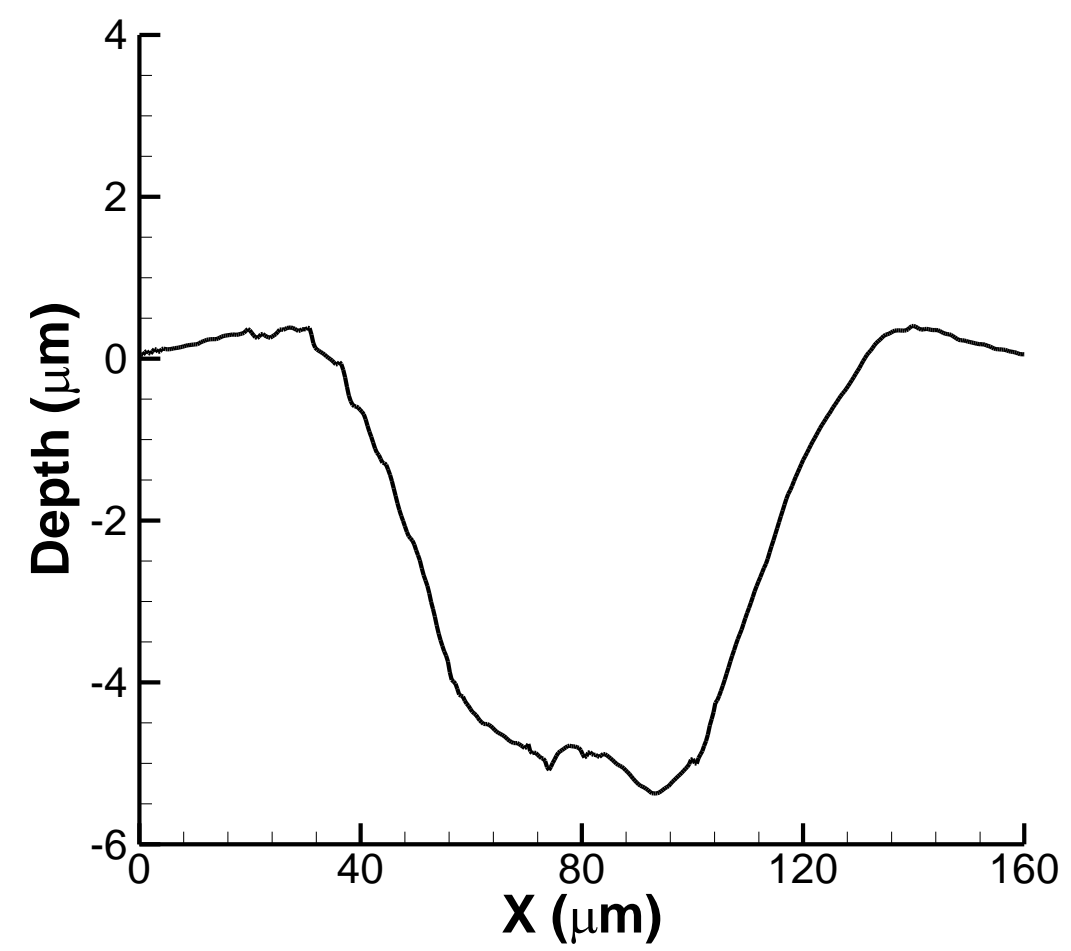

Fig. 22 Ablation profile for copper under laser fluence $36 \mathrm{~J} / \mathrm{cm}^{2}$ (laser beam pulse duration $6 \mathrm{~ns}, 1064 \mathrm{~nm}$, beam diameter $100 \mu \mathrm{m})$

With the MD/SPH model, the liquid ejection from the melt pool could be predicted, as shown in Fig. 23. It can be clearly seen that the melt ejection starts at around $50 \mathrm{~ns}$ and reaches its peak at around $80 \mathrm{~ns}$, which agrees well with the experimental observation shown in Fig. 21. The temperature evolution inside the melt pool and the ejected particle 
is also shown in Fig. 23. The ablation depth is predicted to be around $4.6 \mu \mathrm{m}$ with this $\mathrm{MD} / \mathrm{SPH}$ model, which again agrees well with the experimental data.

The critical point temperature for copper reported in literature is around $8280 \mathrm{~K}$ [35], $8000 \mathrm{~K}$ [36], $7800 \mathrm{~K}[37], 7625 \mathrm{~K}$ [38], $8900 \pm 900 \mathrm{~K}$ [39], $5330 \mathrm{~K}$ [40], $5450 \mathrm{~K}$ [41], $5400 \sim 6000 \mathrm{~K}$ [42-43], $5890 \mathrm{~K}$ [44], $7696 \mathrm{~K}$ [45], and $8650 \mathrm{~K}$ [46]. It should be noted that all the reported values lower than $6000 \mathrm{~K}$ are estimated by Martnyuk [40-44], which are based on the extrapolation of measurement data for discharging a copper wire until electrical explosion.The measurements were made at the initial point of melting and the initial point of electrical explosion, and then the values above normal boiling point were extrapolated. As acknowledged by Martnyuk [44], the error of this estimation could be as high as $15 \%$.All the other reported values are in the range of 7600 to $8900 \mathrm{~K}$. Due to this large discrepancy between the two groups of values, it is imperative to determine which group of the value should be used for this work. Similar to the aluminum case, the critical temperature for copper is also predicted by the MD simulation to be $7900 \pm 20 \mathrm{~K}$ with the method developed in Ref. [29], as shown in Fig. 24. Based on this calculation, the critical temperature of copper should be in the group with higher values. In this work, $\mathrm{T}_{\mathrm{c}}$ value is taken to be $8000 \mathrm{~K}$ since it is in the middle of the reported valuesand also close to the predicted value by MD calculation.

If $0.9 \mathrm{~T}_{\mathrm{c}}$ is used as the criterion for the ablation depth prediction for this phase explosion process, the ablation depth should be around $3.2 \mu \mathrm{m}$ based on the calculation from the HD model, which is almost $40 \%$ lower than the experimental measurement. A further investigation indicates that the predicted ablation depth would increase to around $5.0 \mu \mathrm{m}$ if $0.8 \mathrm{~T}_{\mathrm{c}}$ is used instead as the criterion. 

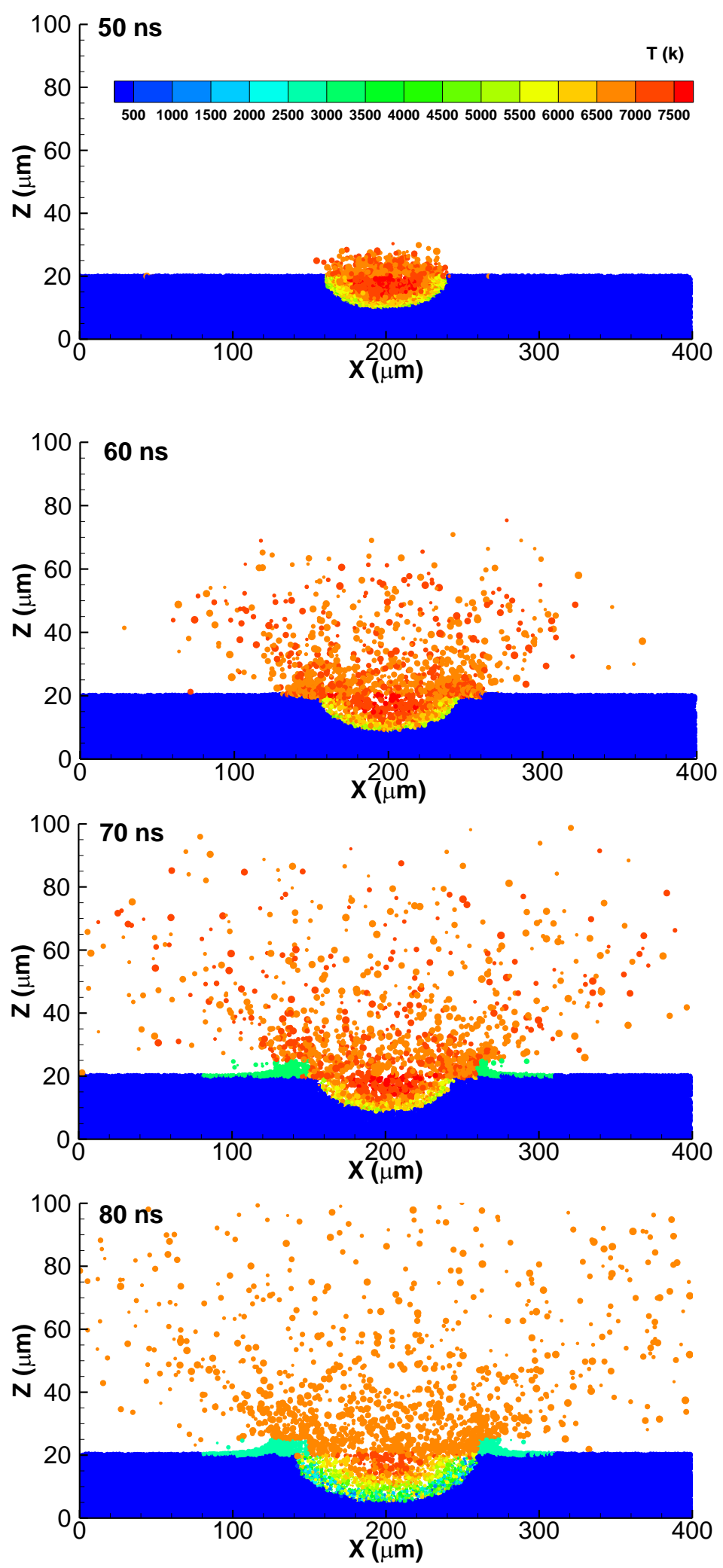

Fig. 23 SPH calculation results showing the melt ejection for copper (laser fluence 36 $\mathrm{J} / \mathrm{cm}^{2}$, wavelength $1064 \mathrm{~nm}$, pulse duration $6 \mathrm{~ns}$, beam diameter $100 \mu \mathrm{m}$ ) 


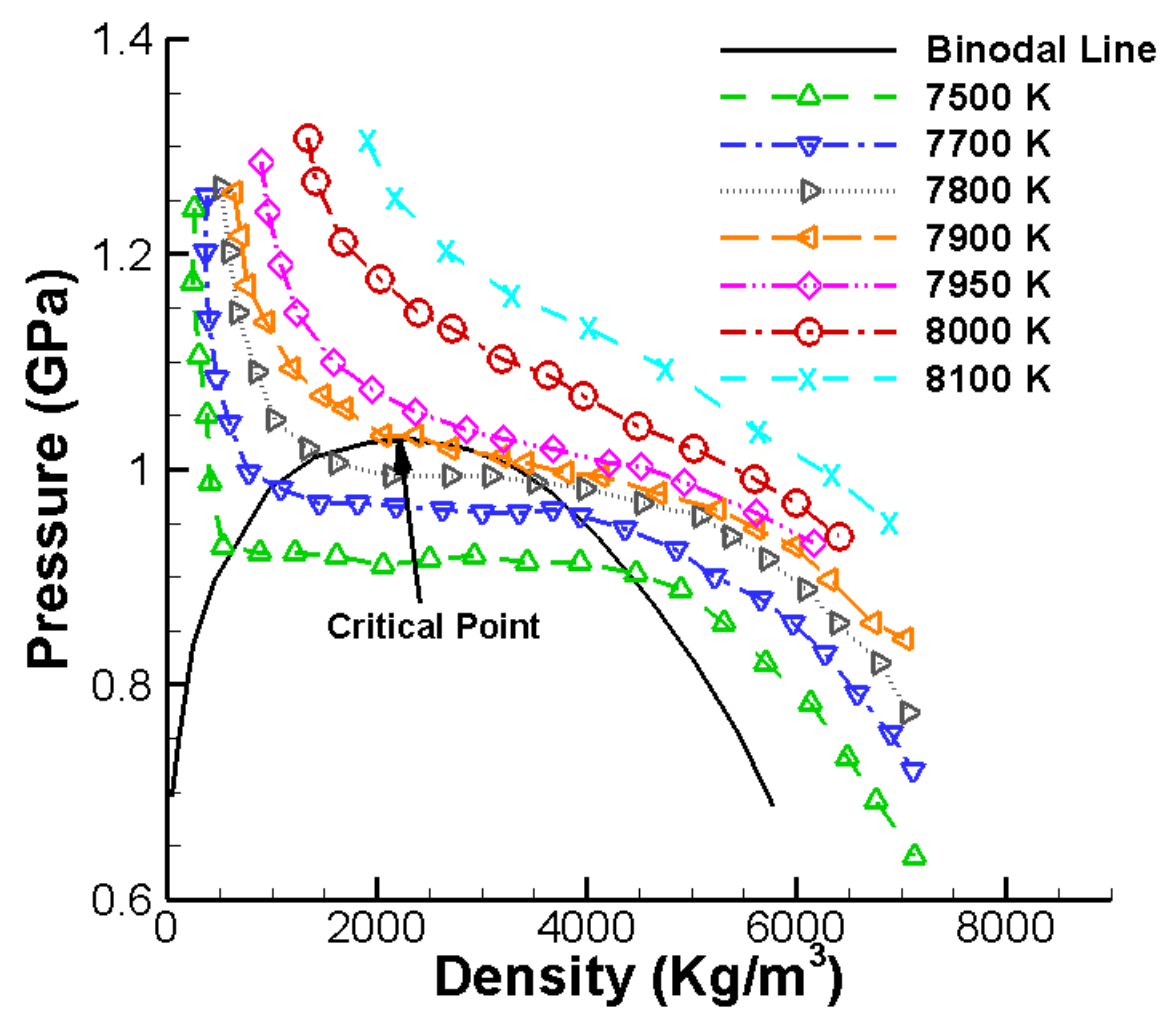

Fig. 24 Calculated isotherms from MD simulation for Copper

More cases with different laser flucences are also tested. The melt ejection is calculated with MD/SPH model and compared with the HD model prediction and experimental data, as seen in Fig. 25. In all the cases shown in Fig. 25, the MD/SPH prediction show better agreement with the experimental data than the HD prediction based on $0.9 \mathrm{Tc}$ as the criterion for the ablation depth prediction. Also if $0.8 \mathrm{~T}_{\mathrm{c}}$ is used as the ablation depth prediction criterion, the HD prediction is closer to the experimental data. If $0.75 \mathrm{~T}_{\mathrm{c}}$ is used as the ablation depth prediction criterion, the HD prediction is overestimating the ablation depth. The comparison shown in Fig. 25 further confirms that the $0.9 \mathrm{~T}_{\mathrm{c}}$ criterion doesn't always work for all materials. At least for copper, 0.75 to 0.8 $\mathrm{T}_{\mathrm{c}}$ might be more appropriate to be used as the ablation depth prediction criterion for the 
HD model in the cases considered in this work. On the other hand, the MD/SPH model could predict the ablation depth and the associated ablation behavior well.

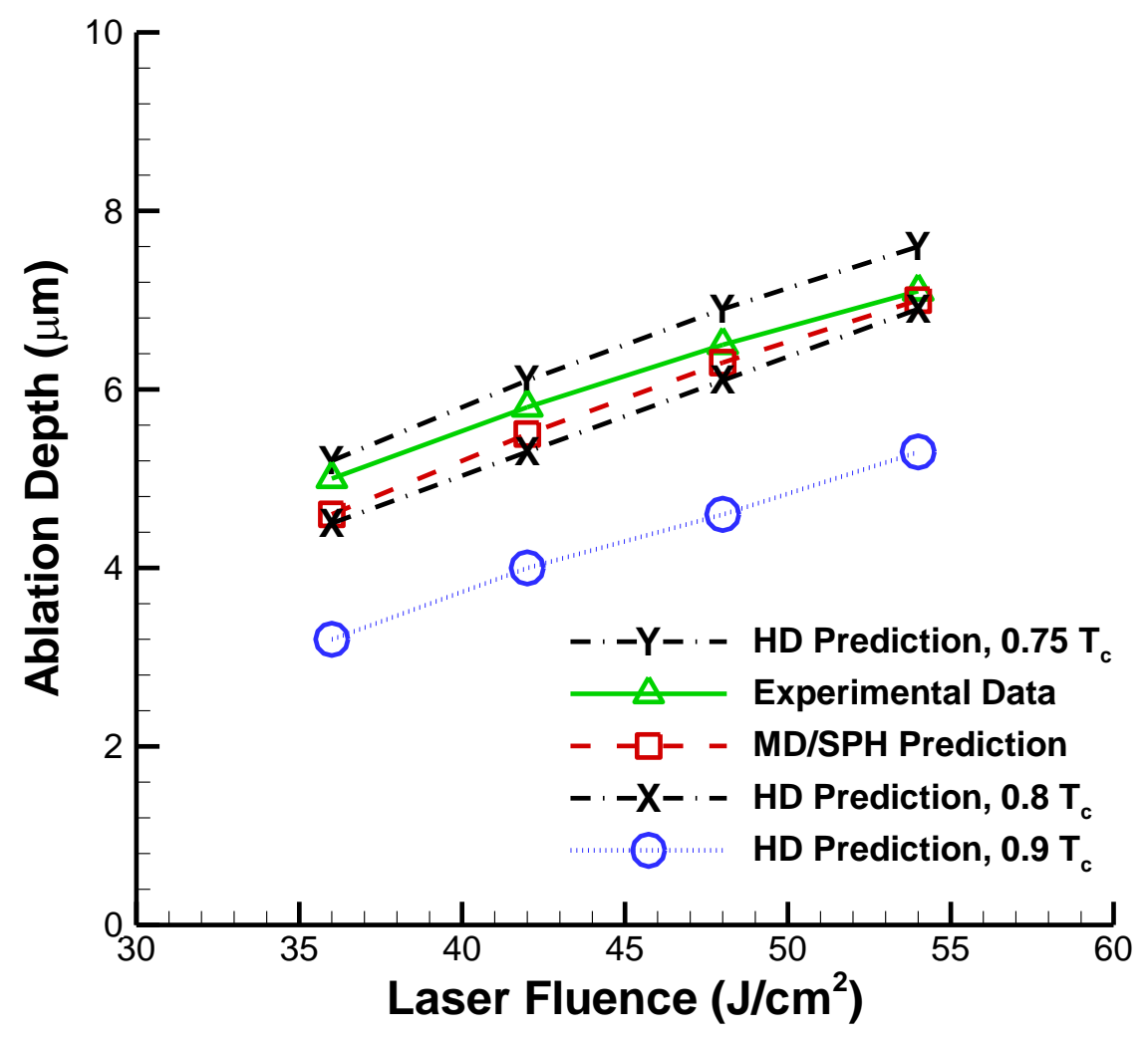

Fig. 25 Comparison of ablation depth under different laser fluences for copper. Simulation data are from the HD model (laser wavelength $1064 \mathrm{~nm}$, pulse duration $6 \mathrm{~ns}$, beam diameter $100.0 \mu \mathrm{m}$, singleshot laser ablation)

Due to the uncertainty of the critical temperature of copper, it is better to investigate the effect of the picked value. If Martnyuk's estimated value (around $5800 \mathrm{~K}$ ) is used in this work, $0.9 \mathrm{~T}_{\mathrm{c}}$ would yield a temperature of $5220 \mathrm{~K}$ and the ablation depth prediction would be over $10 \mu \mathrm{m}$, which would significantly overestimate the copper ablation even under the highest laser fluence shown in Fig. 25. As a result, $0.9 \mathrm{~T}_{\mathrm{c}}$ criterion would not work in all the cases investigated in this work.Similarly, if a much higher value (more than $8500 \mathrm{~K}$ ) is used, the ablation depth would be significantly 
underestimated. This simple analysis indicates that both Martnyuk's estimation and the higher values reported in Ref. [39] and [46]are not applicable for the laser ablation of copper when phase explosion occurs. Based on the value used in this work (8000 K), 0.9 $\mathrm{T}_{\mathrm{c}}$ yields a temperature of $6300 \mathrm{~K}$. To make the $0.9 \mathrm{~T}_{\mathrm{c}}$ criterion work in this temperature, one would need a critical temperature of $7100 \mathrm{~K}$ for copper. To the best knowledge of the authors, there is no critical value reported in this range in the literature. This again verifies that the value used in this work is reasonable for the analysis of phase explosion for copper.

\section{Concluding Remarks}

Nanosecond laser ablation of aluminum and copper with phase explosion was investigated through a multi-scale model and experimental verification. The model prediction of the melt ejection behavior agrees well with the experimental observation in terms of the phase explosion starting time, particle expansion characteristics, and ablation depth. According to the present analysis, the commonly used ablation depth prediction criterion of $0.9 \mathrm{~T}_{\mathrm{c}}$ does not seem to be correct for copper when phase explosion is involved, while it is reasonable for aluminum. In the cases considered in this work, 0.75 0.8 $\mathrm{T}_{\mathrm{c}}$ is found to be a better criterion for copper. The model developed in this work has been shown to provide better capability of predicting the ablation depth and the associated ablation behavior with phase explosion. 
Acknowledgement The authors wish to gratefully acknowledge the financial

supportprovided for this study by the National Science Foundation (Grant No. CMMI-

1030786and CMMI-1300930).

\section{Reference}

[1] Becker, M.F., Brock, J.R., Cai, H., Henneke, D.E., Keto, J.W., Lee, J., Nichols, W.T., Glicksman, H.D., 1998, "Metal Nanoparticles Generated by Laser Ablation", Nanostructured Materials, 10, pp. 853-863.

[2] Kar, A and Mazumder, J., 1994, "Mathematical Model for Laser Ablation to Generate Nanoscale and Submicrometer-size Particles", Physical Review E, 19, pp. 410-419.

[3] Gnedovets, A.G., Gusarov, A.V., Smurov, I., 1999, "A Model for Nanoparticle Synthesis by Pulsed Laser Evaporation", Journal of Physics D: Applied Physics, 32, pp. 2162-2168.

[4] Gnedovets, A.G., Gusarov, A.V., Smurov, I., 2000, "Submicron Particles Synthesis by Laser Evaporation at Low Power Density: ANumerical Analysis", Applied Surface Science, 154-155, pp. 508-513.

[5] Gusarov, A.V., Gnedovets, A.G., Smurov, I., Flamant, G., 2000, "Simulation of Nanoscale Particles Elaboration in Laser-produced Erosive Flow", Applied Surface Science, 154-155, pp. 331-336.

[6] Blair, D., Tillack, M., Zaghloul, M., 2001, "Prediction of Particulate Characteristics in an Expanding Laser Plume", SPIE Conference on Micromachining and Microfabrication, San Francisco, CA, October 2001, Vol. 4557, pp. 139-150.

[7] Brailovsky, A.B., Gaponov, S.V., Luchin, V.I., 1995, "Mechanisms of Melt Droplets and Solid Particle Ejection from ATarget Surface by Pulsed Laser Action", Applied Physics A, 61, pp. 81-86.

[8] Porneala, C., and Willis, D.A., 2006, "Observation of Nanosecond Laser-induced Phase Explosion in Aluminum”, Applied Physics Letters, 89, 211121.

[9] Miotello, A., and Kelly, R., 1999, "Laser-induced Phase Explosion: New Physical Problems when A Condensed Phase Approaches the Thermodynamic Critical Temperature" Applied Physics A, 69, pp. S67-S73.

[10] Liu, Ch., Mao, X., Mao, S., Greif, R., and Russo, R.E., 2005, "Particle Size Dependent Chemistry from Laser Ablation of Brass", Analytical Chemistry, 77 (20), pp. 6687-6691.

[11] Muhammad, N., Rogers, B.D., and Li, L., 2013, "Understanding the Behavior of Pulsed Laser Dry and Wet Micromachining Processes by Multi-phase Smoothed Particle Hydrodynamics (SPH) Modeling", Journal of Physics D: Applied Physics, 46, 095101.

[12] Tong, M., Browne, D.J., 2011, "Smoothed Particle Hydrodynamics Modeling of the Fluid Flow and Heat Transfer in the Weld Pool during Laser Spot Welding", Materials Science and Engineering, 27, pp. 1-7.

[13] Von Allmen, M., 1987, Laser-Beam Interactions with Materials: Physical Principles and Applications, Springer Series in Material Science, Vol. 2, Springer-Verlag, New York. 
[14] Bennett, T.D., Grigoropoulos, C.P., Krajnovich, D.J., 1995, "Near Threshold Laser Sputtering of Gold", Journal of Applied Physics, 77, pp. 849-864.

[15] Hergenroder, R., 2006, "Hydrodynamic Sputtering as a Possible Source for Fractionation in LA-ICP-MS", Journal of Analytical Atomic Spectrometry, 21, pp. 517-524.

[16] Lu, Q., 2003, “Thermodynamic Evolution of Phase Explosion during High-power Nanosecond Laser Ablation", Physical Review E, 67, 016410.

[17] Yoo, J.H., Jeong, S.H., Mao, X.L., Greif, R., and Russo, R.E., 2000, "Evidence for Phase-explosion and Generation of Large Particles during High Power Nanosecond Laser Ablation of Silicon”, Applied Physics Letters, 76, pp. 783-785.

[18] Lu, Q., Mao, S.S., Mao, X.L., and Russo, R.E., 2002, "Delayed Phase Explosion during High-power Nanosecond Laser Ablation of Silicon", Applied Physics Letters, 80, pp. 3072-3074.

[19] Gragossian, A., Tavassoli, S.H., and Shokri, B., 2009, "Laser Ablation of Aluminum from Normal Evaporation to Phase Explosion", Journal of Applied Physics, 105, 103304.

[20] Cao, Y., Zhao, X., and Shin, Y.C., 2013, "Analysis of Nanosecond Laser Ablation of Aluminum with and without Phase Explosion in Air and Water", Journal of Laser Applications, 25 (3), 032002.

[21] Zhigilei, L.V., and Ivanov, D.S., 2005, "Channels of Energy Redistribution in Shortpulse Laser Interactions with Metal Targets", Applied Surface Science, 248(1-4), pp. 433-439.

[22] Allen, M.P., and Tildesley, D.J., 1989, Computer Simulation of Liquids, Clarendon Press, New York.

[23] Manenti, A., 2009, "A Smoothed Particle Hydrodynamics: Basics and Applications", Retrieved from http://www-2.unipv.it/compmech/seminars/manentipres.pdf.

[24] Wu, B. and Shin, Y.C., 2007, “Two Dimensional Hydrodynamic Simulation of High Pressures Induced by High-power Nanosecond Laser-matter Interactions under

Water", Journal of Applied Physics, 101, 103514.

[25]http://lammps.sandia.gov.

[26] Ganzenmuller, G.C, Steinhauser, M.O., and Liedekerke, P.V., 2011, "The Implementation of Smoothed Particle Hydrodynamics in LAMMPS", Retrieved from http://lammps.sandia.gov/doc/USER/sph/SPH_LAMMPS_userguide.pdf.

[27] Baskes, M.I., 1992, "Modified Embedded-atom Potentials for Cubic Materials and Impurities", Physical Review B, 46, pp. 2727-2742.

[28] Linhart, A., Chen, Ch., Vrabec, J., and Hasse, H., 2005, "Thermal Properties of the Metastable Supersaturated Vapor of the Lennard-Jones Fluid", The Journal of Chemical Physics, 122, 144506.

[29] Cheng, Ch. And Xu, X., 2007, "Molecular Dynamics Calculation of Critical Point of Nickel", International Journal of Thermophysics, 28 (1), pp. 9-19.

[30] Morel, V., Bultel, A., and Cheron, B.G., 2009, "The Critical Temperature of Aluminum", International Journal of Thermophysics, 30, pp. 1853-1863.

[31] Bhatt, D., Jasper, A.W., Schultz, N.E., Siepmann, J.I., and Truhlar, D.G., 2006, "Critical Properties of Aluminum", Journal of the American Chemical Society, 128, pp. 4224-4225. 
[32] Sokolowski-Tinten, K., Bialkowski, J., Cavalleri, A., von der Linde, D., Oparin, A., Meyer-terVehn, J., and Ansimov, S.I., 1998, "Transient States of Matter during Short Pulse Laser Ablation", Physical Review Letters, 81, pp. 224-227.

[33] Tavassoli, S.H. and Khalaji, M., 2008, "Laser Ablation of Preheated Copper Samples”, Journal of Applied Physics, 103, 083118.

[34] Liu, C., Mao, X.L., Mao, S.S., Zeng, X., Greif. R., and Russo, R.E., 2004, "Nanosecond and Femtosecond Laser Ablation of Brass: Particulate and ICPMS Measurements", Analytical Chemistry, 76 (2), pp. 379-383.

[35]http://www.efunda.com/materials/elements/element_info.cfm?Element_ID=Cu.

[36] Autrique, D., Chen, Z., Alexiades, V., Bogaerts, A., and Rethfeld, B., 2012, “A Multiphase Model for Pulsed ns-laser Ablation of Copper in an Ambient Gas", AIP Conference Proceedings, 1464, pp. 648-659

[37] Sugioka, K. and Cheng, Y., 2013, Ultrafast Laser Processing: From Micro- to Nanoscale, CRC Press, Boca Raton, FL.

[38] Young, D.A. and Alder, B.J., 1971, "Critical Point of Metals from the Van Der Waals Equation of State", Physical Review A, 3 (1), pp. 364-371.

[39] Cahill, J.A. and Kirshenbaum, A.D., 1962, "The Density of Liquid Copper from Its Melting Point $\left(1356{ }^{\circ} \mathrm{K}\right)$ to $2500{ }^{\circ} \mathrm{K}$ and an Estimation of Its Critical Constants", Journal of Physical Chemistry, 66 (6), pp. 1080-1082.

[40] Martynyuk, M.M., 1977, "The Critical Parameters of Metals I, Critical Temperature", Russian Journal of Physical Chemistry, 51, 705-706.

[41] Martynyuk, M.M., 1977, "Phase Explosion of a Metastable Fluid", Combustion, Explosion and Shock Waves, 13 (2), pp. 178-191.

[42] Kelly, R. and Miotello, A., 1996, "Comments on Explosive Mechanisms of Laser Sputtering”, Applied Surface Science, 96-98, pp. 205-215.

[43] Martyuyuk, M.M., 1983, "Critical Constants of Metals”, Russian Journal of Physical Chemistry, 57, pp. 494-501.

[44] Martynyuk, M.M., 1992, "Superheating of Solid and Liquid Metals in the Process of Pulse Heating", Thermochimica Acta, 206, pp. 55-60.

[45] Hess, H., 1998, "Critical Data and Vapor Pressures of Aluminum and Copper", Zeitschrift fur Metallkunde, 89, pp. 388-393.

[46] Singh, J.K., Adhikari, J. and Kwak, S.K., 2006, "Vapor-liquid Phase Coexistence Curves for Morse Fluids”, Fluid Phase Equilibria, 248, pp. 1-6. 


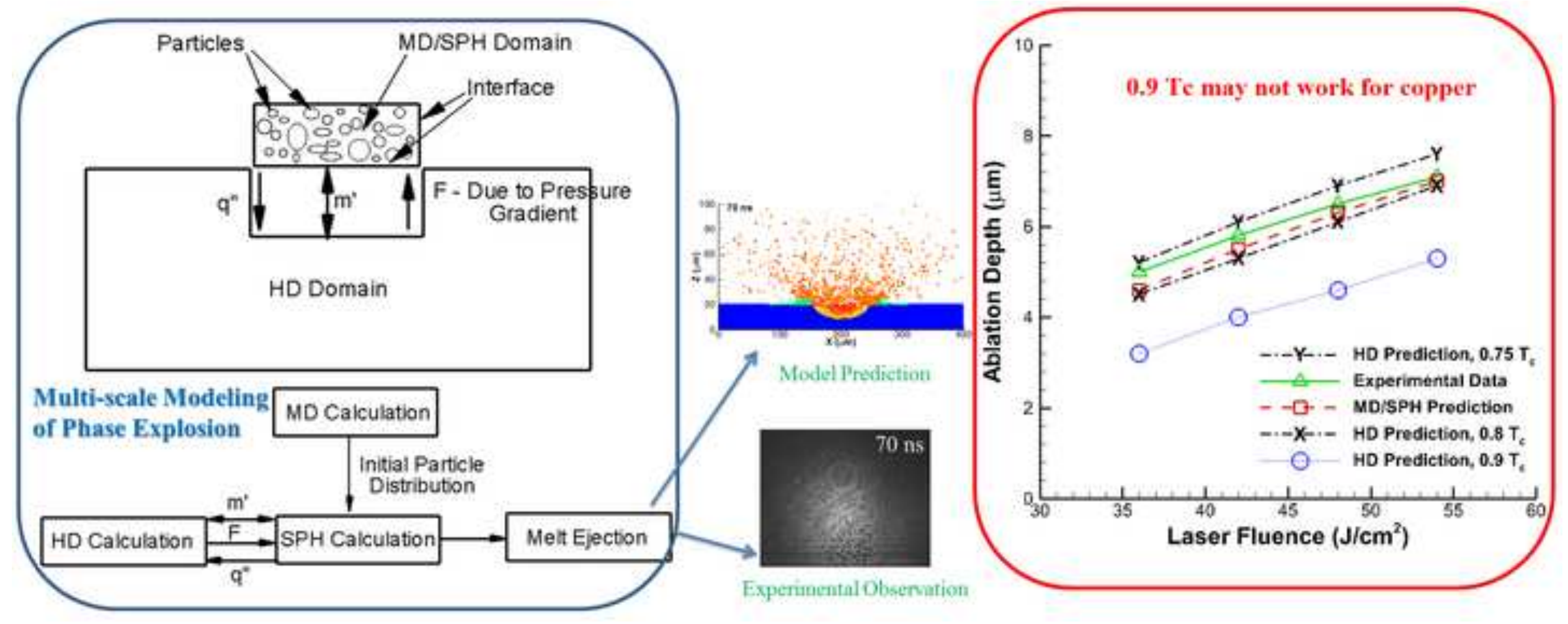

